\title{
Present status of eriophyoid mites in Thailand
}

\author{
Angsumarn CHANDRAPATYA ${ }^{1}$, Ploychompoo KONVIPASRUANG $^{2}$ and James W. AMRINE ${ }^{3}$ \\ ${ }^{1}$ Department of Entomology, Faculty of Agriculture, Kasetsart University, 50 Ngam Wong Wan \\ Road, Chatuchak, Bangkok 10900, Thailand \\ ${ }^{2}$ Plant Protection Research and Development Office, Department of Agriculture, Paholyothin Road, \\ Chatuchak, Bangkok 10900, Thailand \\ ${ }^{3}$ Division of Plant and Soil Sciences, College of Agriculture and Forestry, West Virginia University, \\ P. O. Box 6108, Morgantown, WV 26506-6108, USA
}

\begin{abstract}
One of the common groups of phytophagous mites encountered on various plants in Thailand is that of the eriophyoid mites, which can be found on agricultural, horticultural, ornamental, and medicinal plants, including fruit and forest trees. Because there is a paucity of information on eriophyoid taxonomy in Thailand, where the host plants are so diverse, there is a need to investigate the presence of these tiny creatures - especially those species that can be harmful to economic crops. Here, the taxonomy of the eriophyoid mites in the collection of the first author was revised, together with the taxonomy of those reported by other researchers. To date, a total of 215 species of eriophyoid mites have been recorded from Thailand. The family Eriophyidae comprises 157 species, whereas only 58 species are reported in the family Diptilomiopidae. These mites are found on 161 plant species under 60 host plant families; they are relatively more numerous ( $>10$ species) on plants in the families Fabaceae, Poaceae, Moraceae, Sapindaceae, Rubiaceae, Anacardiaceae, Myrtaceae, and Euphorbiaceae. Fortunately, only a few species are considered to be pests of our economic crops. Eight new genera were assigned as new names for those mites previously identified by the first author and Professor Jan Boczek. Another 8 new genera and 10 new species were also added for unidentified eriophyoid mites in this collection where Diptilomiopus phylanthi (Chandrapatya, 1992), Liparus eugeniae Boczek, 1996 and Tegolophus bangkokensis Keifer, 1978 were removed from the list bringing the total number of eriophyoid mites found in Thailand to 222 species. Four replacement names were proposed in this paper; Cosella trangii Chandrapatya, Konvipasruang \& Amrine, 2016 for Cosella longani (Boczek \& Chandrapatya, 2000) since it is too close to Cosella longana Huang, Huang \& Wang, 1996; Phyllocoptes takhoppae Chandrapatya, Konvipasruang \& Amrine, 2016 for Phyllocoptes indicus Boczek \& Chandrapatya, 2000 since it is too close to Phyllocoptes indicae Keifer, 1975; Aculops
\end{abstract}

Tetsuo Gotoh and DeMar TAYLOR (eds.), Acarology XIV: Proceedings of the International Congress. Journal of Acarological Society of Japan 25 (Suppl. 1): 1-192.

* Corresponding author: e-mail: agramc@ku.ac.th; tel: 66-2-942-8350; fax: 66-2-561-4882

This research was supported by the Faculty of Agriculture and the Center for Advanced Studies for Agriculture and Food, KU Institute for Advanced Studies, Kasetsart University, under the Higher Education Research Promotion and National Research University Project of Thailand, Office of the Higher Education Commission and the Thailand Research Fund (TRF Senior Research Scholar \#RTA4880006).

DOI: 10.2300 /acari.25.Suppl_83 
chonburi Chandrapatya, Konvipasruang \& Amrine, 2016 for Aculops desmodii Chandrapatya \& Boczek, 2000 preoccupied by Aculops desmodii (Keifer, 1964) and Diptilomiopus sakaena Chandrapatya, Konvipasruang \& Amrine, 2016 for Diptilomiopus combretae (Chandrapatya \& Boczek, 2002) preoccupied by Diptilomiopus combretae Wei \& Lu, 2001.

Key words: eriophyoid mite, Eriophyidae, Diptilomiopidae, taxonomy, Thailand

\section{INTRODUCTION}

Eriophyid mites are minute, and some of them cause severe damage to host plants. For example, Aceria litchii (Keifer, 1943) induces the production of erineum galls on the leaf undersurfaces of susceptible plants in many countries, such as Taiwan (Huang et al. 1989), Japan (Kadono 1994), India (Haque 2000), and Florida (Florida Department of Agriculture and Consumer Services 2006), whereas Phyllocoptruta oleivora (Ashmead, 1879) causes citrus fruit and leaf rusting, silvering, bronzing, and blackening, resulting in low fruit quality, especially in major orange-producing areas such as Florida (Morse et al. 1996) and mainland China (Hong et al. 2006).

In Thailand, as in other regions of the world, eriophyoid mites infest a number of agricultural, horticultural, ornamental, medicinal, and forest plants. However, taxonomic work on Thai eriophyoid mites is still limited. H. H. Keifer is considered the pioneer of eriophyoid mite study in Thailand and published details of several new genera and species in his Keifer C series numbers 9 to 15 (Keifer 1974, 1975a, b, 1976, 1977a, b, 1978). At the time, 35 species of Thai eriophyoid mites had been reported in the "Eriophyid Mites of Thailand;" 27 species were new to science (Keifer and Knorr 1978). Later, in the late 1980 to early $2000 \mathrm{~s}$, Chandrapatya and Boczek published a series on eriophyoid mites found in Thailand; more than 160 new species were reported. To date, approximately 206 eriophyid species are known from Thailand; all except 3 of these are endemic (Xue and Zhang 2009).

However, there are still some errors in the identification of mites previously described in the literature. Hence, there is a need to re-examine the taxonomic characters of all of these previously described mites; moreover, the scientific names need to be adjusted according to the generic key provided by Amrine et al. (2003) for the world genera of eriophyoid mites.

Because eriophyoid mites tend to become pests of economic crops and various plants in many countries, there is still a need to investigate the present status of these mites in Thailand. Therefore, a study of the taxonomy of eriophyoid mites in Thailand is required to provide basic information before control measures can be effectively applied. At this stage, the work has relied on the available literature and slide specimens collected by the first author and deposited in the Museum of the Department of Entomology, Kasetsart University. In addition, researchers at the Plant Protection Research and Development Office, Department of Agriculture, Ministry of Agriculture and Cooperatives, Thailand, collected several eriophyoid mites infesting crops in many of Thailand's provinces. The distributions of these specimens were added to the list of host plants and locations. The information gained from this study will further our understanding of the status of eriophyoid mites in Thailand. 


\section{MATERIALS AND METHODS}

Mite samples were soaked in Keifer I solution until completely cleared. They were then mounted on microscopic slides; Keifer III solution was used as a mounting medium (Keifer 1954). All microscopic slides containing eriophyoid mites were examined under an Olympus BX 43 phase-contrast microscope. Keys to the World Genera of Eriophyoidea (Acari: Prostigmata), prepared by Amrine et al. (2003), were used to identify the mites. Information appearing in "Catalog of the Eriophyoidea (Acarina: Prostigmata) of the World" (Amrine and Stasny 1994) and "Eriophyoid Mites (Acari: Prostigmata) in Southeast Asia: a Synopsis of 104 Genera, with an Illustrated Key to Genera and Checklist of Species" (Xue and Zhang 2009) were also used for proper identification. Type specimens were deposited in the collection of the Department of Entomology, Kasetsart University, Bangkok, Thailand.

\section{RESULTS AND DISCUSSION}

Eriophyoid mites infested a number of host plants, ranging from agricultural to horticultural, ornamental, medicinal, and forest plants collected from 46 out of 76 provinces in Thailand. Data on a total of 215 eriophyoid mites were available from the literature; eight new genera and ten new species were added for the unidentified mites in this study whereas Diptilomiopus phylanthi (Chandrapatya, 1992), Liparus eugeniae Boczek, 1996, and Tegolophus bangkokensis Keifer, 1978 were removed from the list since they were treated as synonyms of Diptilomiopus securinegus (Boczek, 1992), Lipacarus eugeniae (Boczek, 1992), and Phaicus bambusae (ChannaBassavanna, 1966), respectively. Therefore, a list of 222 eriophyoid mites of Thailand is reported in Table 1. The majority of these species $(>160)$ were identified by the first author and co-author(s), and another 35 species were reported by Keifer and Knorr (1978). About $72.07 \%$ of eriophyoid mites (160 species) are classified in the family Eriophyidae and only $27.93 \%$ (62 species) belong to the family Diptilomiopidae. Eriophyoid mites in the family Phytoptidae are not found in Thailand.

A total of 161 plant species within 60 host plant families harbored eriophyoid mites. More than 10 mite species were found on some plant families, such as the Fabaceae, Poaceae, Moraceae, Sapindaceae, Rubiaceae, Rutaceae, Anacardiaceae, Myrtaceae, and Euphorbiaceae (Table 1). In general, the eriophyoid mite is a monophagous feeder. However, some eriophyoid mites in Thailand were found feeding on more than two host species: for example, Neocatarhinus namtai Konvipasruang, Chandrapatya \& Amrine, 2012 infested Bambusa ventricosa McClure, Bambusa vulgaris Schrad. ex J. C. Wendl., Dendrocalamus asper (Schult. \& Schult. f.) Backer ex K. Heyne, and Melocanna humilis Roep. ex Trin.; and Kolacarus bambusae Boczek, 1998 fed on Bambusa bambos (L.) Voss, Bambusa blumeana Schult. \& Schult. f., Bambusa multiplex (Lour.) Raeusch. ex Schult. \& Schult. f., Bambusa ventricosa, Bambusa vulgaris, and Dendrocalamus latiflorus Munro.

Mite species that infested economic plants and were responsible for yield loss or degradation of plant production included Aceria tulipae (Keifer, 1938) on garlic, Aceria litchii (Keifer) on 
Table 1. List of eriophyoid mites and associated host plants found in Thailand

\begin{tabular}{|c|c|c|c|c|}
\hline Eriophyoid mite & Host plant & Host family & $\begin{array}{l}\text { Remarks } \\
\text { onmite }\end{array}$ & Reference for mite \\
\hline $\begin{array}{l}\text { Abacarus asperi } \\
\text { (Chandrapatya \& Boczek, } \\
\text { 2002) }\end{array}$ & $\begin{array}{l}\text { Dendrocalamus asper } \\
\text { (Schult. \& Schult.f.) Backer } \\
\text { ex K. Heyne }\end{array}$ & $\begin{array}{l}\text { Poaceae, } \\
\text { Bambuseae }\end{array}$ & - & $\begin{array}{l}\text { Chandrapatya \& Boczek } \\
\text { 2002b } \\
\text { Konvipasruang et al. 2012a }\end{array}$ \\
\hline $\begin{array}{l}\text { Abacarus euphoriae Keifer, } \\
1975\end{array}$ & $\begin{array}{l}\text { Dimocarpus longan Lour. } \\
\text { ssp. longan var. longan } \\
\text { Litchi chinensis Sonn. }\end{array}$ & Sapindaceae & - & $\begin{array}{l}\text { Keifer 1975b } \\
\text { Chandrapatya \& Boczek } \\
1996\end{array}$ \\
\hline $\begin{array}{l}\text { Abacarus klaengensis } \\
\text { Konvipasruang, } \\
\text { Chandrapatya \& Amrine, } \\
2012\end{array}$ & $\begin{array}{l}\text {-Bambusa sp. } \\
\text {-Bambusa multiplex (Lour.) } \\
\text { Raeusch. ex Schult. \& } \\
\text { Schult.f. }\end{array}$ & $\begin{array}{l}\text { Poaceae, } \\
\text { Bambuseae }\end{array}$ & - & $\begin{array}{l}\text { Boczek \& Chandrapatya } \\
\text { 1998a } \\
\text { Konvipasruang et al. 2012a }\end{array}$ \\
\hline $\begin{array}{l}\text { Abacarus oryzae Keifer, } \\
1963\end{array}$ & Oryza sativa $\mathrm{L}$. & Poaceae & - & Keifer 1963 \\
\hline $\begin{array}{l}\text { Abacarus pennatus } \\
\text { Chandrapatya, } 1991\end{array}$ & $\begin{array}{l}\text { Acacia pennata (L.) Willd. } \\
\text { ssp. insuavis (Lace) I. C. } \\
\text { Nielsen }\end{array}$ & Fabaceae & - & $\begin{array}{l}\text { Chandrapatya \& Boczek } \\
\text { 1991b }\end{array}$ \\
\hline $\begin{array}{l}\text { Abacarus sacchari } \\
\text { ChannaBasavanna, } 1966\end{array}$ & -Saccharum officinarum $\mathrm{L}$. & Poaceae & - & ChannaBasavanna1966 \\
\hline $\begin{array}{l}\text { Acalitus odoratus Keifer, } \\
1970\end{array}$ & $\begin{array}{l}\text { Chromolaena odorata }(\mathrm{L} .) \\
\text { R. M. King \& H. Robinson }\end{array}$ & Asteraceae & - & $\begin{array}{l}\text { Amrine \& Stasny } 1994 \\
\text { Keifer } 1970\end{array}$ \\
\hline $\begin{array}{l}\text { Acarhis lepisanthis Keifer, } \\
1975\end{array}$ & $\begin{array}{l}\text { Lepisanthes rubiginosa } \\
\text { (Roxb.) Leenth. }\end{array}$ & Sapindaceae & - & Keifer 1975b \\
\hline $\begin{array}{l}\text { Acarhis siamensis Boczek \& } \\
\text { Chandrapatya, } 2000\end{array}$ & $\begin{array}{l}\text { Rauwenhoffia siamensis R. } \\
\text { Scheff. }\end{array}$ & Annonaceae & - & $\begin{array}{l}\text { Boczek \& Chandrapatya } \\
\text { 2000a }\end{array}$ \\
\hline $\begin{array}{l}\text { Aceria binaria (Keifer, } \\
\text { 1977) }\end{array}$ & $\begin{array}{l}\text { Peltophorum pterocarpum } \\
\text { (DC.) Backer ex K. Heyne }\end{array}$ & Fabaceae & emendation & Keifer 1997b \\
\hline $\begin{array}{l}\text { Aceria dioscoridis (Soliman } \\
\& \text { Abou-Awad, 1977) }\end{array}$ & -Pluchea indica (L.) Less. & Asteraceae & - & $\begin{array}{l}\text { Soliman \& Abou-Awad } \\
1977 \\
\text { Amrine \& Stasny } 1994 \\
\end{array}$ \\
\hline Aceria ghanii Keifer, 1966 & -Ziziphus mauritiana Lam. & Rhamnaceae & - & Keifer 1966b \\
\hline Aceria litchii (Keifer, 1943) & -Litchi chinensis Sonn. & Sapindaceae & - & $\begin{array}{l}\text { Keifer } 1943 \\
\text { Amrine \& Stasny } 1994\end{array}$ \\
\hline $\begin{array}{l}\text { Aceria longana Boczek \& } \\
\text { Knihinicki, } 1998\end{array}$ & $\begin{array}{l}\text { Dimocarpus longan Lour. } \\
\text { ssp. longan var. longan }\end{array}$ & Sapindaceae & - & Boczek \& Knihinicki 1998 \\
\hline $\begin{array}{l}\text { Aceria mangiferae Sayed, } \\
1946\end{array}$ & - Mangifera indica $\mathrm{L}$. & Anacardiaceae & - & Sayed 1946 \\
\hline $\begin{array}{l}\text { Aceria neopaederiae } \\
\text { Konvipasruang, } \\
\text { Chandrapatya \& Amrine, } \\
2012\end{array}$ & -Paederia foetida $\mathrm{L}$. & Rubiaceae & - & $\begin{array}{l}\text { Konvipasruang et al. } \\
2012 \mathrm{~b}\end{array}$ \\
\hline $\begin{array}{l}\text { Aceria sandorici (Nalepa, } \\
\text { 1914) }\end{array}$ & $\begin{array}{l}\text { Sandoricum koetjape (Burm. } \\
\text { f.) Merr. }\end{array}$ & Meliaceae & - & $\begin{array}{l}\text { Nalepa, } 1914 \\
\text { Amrine \& Stasny } 1994\end{array}$ \\
\hline $\begin{array}{l}\text { Aceria sarmentosae } \\
\text { Chandrapatya, } 1992\end{array}$ & $\begin{array}{l}\text { Azima sarmentosa (Blume) } \\
\text { Benth. \& Hook.f. }\end{array}$ & Salvadoraceae & - & $\begin{array}{l}\text { Boczek \& Chandrapatya } \\
\text { 1992a }\end{array}$ \\
\hline Aceria tulipae (Keifer, 1938) & $\begin{array}{l}\text {-Allium sativum } \mathrm{L} . \\
\text {-Allium ascalonicum } \mathrm{L} \text {. }\end{array}$ & Alliaceae & - & $\begin{array}{l}\text { Keifer } 1938 \\
\text { Keifer } 1944\end{array}$ \\
\hline $\begin{array}{l}\text { Acerimina terminaliae } \\
\text { Keifer, } 1974\end{array}$ & - Terminalia catappa $\mathrm{L}$. & Combretaceae & - & Keifer 1974 \\
\hline $\begin{array}{l}\text { Aculops albizi (Boczek \& } \\
\text { Chandrapatya, 2000) }\end{array}$ & -Albizia lebbeck (L.) Benth. & Fabaceae & $\begin{array}{l}\text { new } \\
\text { combination }\end{array}$ & $\begin{array}{l}\text { Boczek \& Chandrapatya } \\
\text { 2000e }\end{array}$ \\
\hline $\begin{array}{l}\text { Aculops azimae } \\
\text { (Chandrapatya, 1993) }\end{array}$ & $\begin{array}{l}\text { Azima sarmentosa (Blume) } \\
\text { Benth. \& Hook.f. }\end{array}$ & Salvadoraceae & - & $\begin{array}{l}\text { Chandrapatya \& Boczek } \\
\text { 1993a } \\
\text { Amrine \& Stasny } 1996\end{array}$ \\
\hline $\begin{array}{l}\text { Aculops cannae (Boczek, } \\
\text { 1992) }\end{array}$ & Canna orientalis Roscoe & Cannaceae & $\begin{array}{l}\text { new } \\
\text { combination }\end{array}$ & $\begin{array}{l}\text { Boczek \& Chandrapatya } \\
\text { 1992b }\end{array}$ \\
\hline
\end{tabular}




\begin{tabular}{|c|c|c|c|c|}
\hline Eriophyoid mite & Host plant & Host family & $\begin{array}{c}\text { Remarks } \\
\text { onmite }\end{array}$ & Reference for mite \\
\hline $\begin{array}{l}\text { Aculops caricae Keifer, } \\
1977\end{array}$ & Carica papaya $\mathrm{L}$. & Caricaceae & & $\begin{array}{l}\text { Keifer 1977a } \\
\text { Boczek \& Chandrapatya } \\
\text { 1996b }\end{array}$ \\
\hline $\begin{array}{l}\text { Aculops chonburi } \\
\text { Chandrapatya, } \\
\text { Konvipasruang \& Amrine, } \\
2016\end{array}$ & $\begin{array}{l}\text { Desmodium gangeticum (L.) } \\
\text { DC. }\end{array}$ & Fabaceae & $\begin{array}{l}\text { replacement } \\
\text { name }\end{array}$ & $\begin{array}{l}\text { Chandrapatya \& Boczek } \\
\text { 2000a }\end{array}$ \\
\hline $\begin{array}{l}\text { Aculops cratevi Boczek \& } \\
\text { Chandrapatya, } 2000\end{array}$ & $\begin{array}{l}\text { Crateva adansonii DC. } \\
\text { subsp. trifoliata (Roxb.) } \\
\text { Jacobs } \\
\text { Crateva roxburghii } \text { R.Br. }\end{array}$ & Capparidaceae & - & $\begin{array}{l}\text { Boczek \& Chandrapatya } \\
2000 \mathrm{~b}\end{array}$ \\
\hline $\begin{array}{l}\text { Aculops glabrati Boczek \& } \\
\text { Chandrapatya, } 2000\end{array}$ & Vitex glabrata $\mathrm{R}$. Br. & Labiaceae & - & $\begin{array}{l}\text { Boczek \& Chandrapatya } \\
\text { 2000b }\end{array}$ \\
\hline Aculops knorri Keifer, 1976 & $\begin{array}{l}\text {-Lepisanthes rubiginosa } \\
\text { (Roxb.) Leenth. }\end{array}$ & Sapindaceae & - & Keifer 1976 \\
\hline $\begin{array}{l}\text { Aculops pelekassi (Keifer, } \\
\text { 1959) }\end{array}$ & Citrus sp & Rutaceae & - & $\begin{array}{l}\text { Keifer } 1959 \\
\text { Keifer } 1966 c\end{array}$ \\
\hline $\begin{array}{l}\text { Aculus dipterocarpi } \\
\text { (Boczek, 1993) }\end{array}$ & $\begin{array}{l}\text { Dipterocarpus alatus Roxb. } \\
\text { ex G. Don }\end{array}$ & Dipterocarpaceae & - & $\begin{array}{l}\text { Chandrapatya \& Boczek } \\
\text { 1993b } \\
\text { Chandrapatya et al. 2014a }\end{array}$ \\
\hline $\begin{array}{l}\text { Aculus menoni } \\
\text { ChannaBasavanna, } 1966\end{array}$ & - Moringa oleifera $\mathrm{Lam}$. & Moringaceae & - & ChannaBasavanna 1966 \\
\hline $\begin{array}{l}\text { Aculus morindai } \\
\text { (Chandrapatya, 1996) }\end{array}$ & - Morinda citrifolia $\mathrm{L}$. & Rubiaceae & $\begin{array}{l}\text { new } \\
\text { combination }\end{array}$ & $\begin{array}{l}\text { Boczek \& Chandrapatya } \\
\text { 1996b }\end{array}$ \\
\hline $\begin{array}{l}\text { Aculus tetraspermae } \\
\text { (Chandrapatya, 1992) }\end{array}$ & -Salix tetrasperma Roxb. & Salicaceae & $\begin{array}{l}\text { new } \\
\text { combination }\end{array}$ & $\begin{array}{l}\text { Boczek \& Chandrapatya } \\
\text { 1992a }\end{array}$ \\
\hline $\begin{array}{l}\text { Afemurseta acuminatae } \\
\text { (Boczek \& Chandrapatya, } \\
2000 \text { ) }\end{array}$ & -Musa acuminata Colla & Musaceae & - & $\begin{array}{l}\text { Boczek \& Chandrapatya } \\
\text { 2000e } \\
\text { Tan et al. } 2014 \\
\text { Chandrapatya et al. } 2015\end{array}$ \\
\hline $\begin{array}{l}\text { Areekulus engeniae } \\
\text { Chandrapatya, } 1998\end{array}$ & Syzygium jambos L. Alston & Myrtaceae & - & $\begin{array}{l}\text { Boczek \& Chandrapatya } \\
\text { 1998b }\end{array}$ \\
\hline $\begin{array}{l}\text { Asetacus elaeocarpi } \\
\text { Chandrapatya \& Boczek, } \\
2000\end{array}$ & $\begin{array}{l}\text { Elaeocarpus lanceifolius } \\
\text { Roxb. }\end{array}$ & Elaeocarpaceae & - & $\begin{array}{l}\text { Chandrapatya \& Boczek } \\
\text { 2000e }\end{array}$ \\
\hline $\begin{array}{l}\text { Bangkokarus diospyrosis } \\
\text { (Chandrapatya, 1991) }\end{array}$ & -Diospyros rhodocalyx Kurz & Ebenaceae & $\begin{array}{l}\text { new genus, } \\
\text { new } \\
\text { combination }\end{array}$ & $\begin{array}{l}\text { Chandrapatya\& Boczek } \\
\text { 1991cChandrapatya et al. } \\
2015\end{array}$ \\
\hline $\begin{array}{l}\text { Bangkophyes fici Boczek \& } \\
\text { Chandrapatya, } 2001\end{array}$ & Ficus hispida L.f. & Moraceae & - & $\begin{array}{l}\text { Boczek \& Chandrapatya } \\
2001\end{array}$ \\
\hline $\begin{array}{l}\text { Bangphracarus } \\
\text { alatus Chandrapatya, } \\
\text { Konvipasruang \& Amrine, } \\
2014\end{array}$ & $\begin{array}{l}\text {-Dipterocarpus alatus Roxb. } \\
\text { ex G. Don }\end{array}$ & Dipterocarpaceae & - & Chandrapatya et al. 2014a \\
\hline $\begin{array}{l}\text { Bischofius anthocephali } \\
\text { (Boczek \& Chandrapatya, } \\
\text { 2000) }\end{array}$ & $\begin{array}{l}\text {-Neolamarckia cadamba } \\
\text { (Roxb.) Bosser }\end{array}$ & Rubiaceae & $\begin{array}{l}\text { new } \\
\text { combination }\end{array}$ & $\begin{array}{l}\text { Boczek \& Chandrapatya } \\
\text { 2000d }\end{array}$ \\
\hline $\begin{array}{l}\text { Bischofius kanchanaburi } \\
\text { Boczek \& Chandrapatya, } \\
2000\end{array}$ & - Bischofia javanica Blume & Phyllanthaceae & - & $\begin{array}{l}\text { Boczek \& Chandrapatya } \\
\text { 2000d }\end{array}$ \\
\hline $\begin{array}{l}\text { Brachendus dimocarpi } \\
\text { Boczek, } 1996\end{array}$ & $\begin{array}{l}\text { Dimocarpus longan Lour. } \\
\text { ssp. longan var. longan }\end{array}$ & Sapindaceae & - & $\begin{array}{l}\text { Chandrapatya \& Boczek } \\
1996\end{array}$ \\
\hline $\begin{array}{l}\text { Calacarus allamandae } \\
\text { Boczek \& Chandrapatya, } \\
1989\end{array}$ & - Allamanda cathartica L. & Apocynaceae & - & $\begin{array}{l}\text { Boczek \& Chandrapatya } \\
\text { 1989b }\end{array}$ \\
\hline
\end{tabular}




\begin{tabular}{|c|c|c|c|c|}
\hline Eriophyoid mite & Host plant & Host family & $\begin{array}{c}\text { Remarks } \\
\text { onmite }\end{array}$ & Reference for mite \\
\hline $\begin{array}{l}\text { Calacarus alocasiae Keifer, } \\
1978\end{array}$ & Alocasia sp. & Araceae & - & Keifer 1978 \\
\hline $\begin{array}{l}\text { Calacarus celosiae Boczek } \\
\& \text { Chandrapatya, } 1989\end{array}$ & - Celosia argentea $\mathrm{L}$. & Amaranthaceae & - & $\begin{array}{l}\text { Boczek \& Chandrapatya } \\
1989 \text { b }\end{array}$ \\
\hline $\begin{array}{l}\text { Calacarus cerberi } \\
\text { (Chandrapatya \& Boczek, } \\
\text { 2001) }\end{array}$ & - Cerbera odollam Gaertn & Apocynaceae & $\begin{array}{l}\text { new } \\
\text { combination }\end{array}$ & $\begin{array}{l}\text { Chandrapatya \& Boczek } \\
\text { 2001c }\end{array}$ \\
\hline $\begin{array}{l}\text { Calacarus } \\
\text { channabasavannae } \\
\text { Lakkundi, } 1974\end{array}$ & $\begin{array}{l}\cdot \text { Annona squamosa } \mathrm{L} \text {. } \\
\cdot \text { Ricinus communis } \mathrm{L} \text {. }\end{array}$ & $\begin{array}{l}\text { Annonaceae } \\
\text { Euphorbiaceae }\end{array}$ & - & Lakkundi 1974 \\
\hline $\begin{array}{l}\text { Calacarus duranti } \\
\text { (Chandrapatya \& Boczek, } \\
\text { 2001) }\end{array}$ & - Duranta erecta $\mathrm{L}$. & Verbenaceae & $\begin{array}{l}\text { new } \\
\text { combination }\end{array}$ & $\begin{array}{l}\text { Chandrapatya \& Boczek } \\
\text { 2001c }\end{array}$ \\
\hline $\begin{array}{l}\text { Calacarus lantanae Boczek } \\
\& \text { Chandrapatya, } 1989\end{array}$ & - Lantana camara $\mathrm{L}$. & Verbenaceae & - & $\begin{array}{l}\text { Boczek \& Chandrapatya } \\
\text { 1989a }\end{array}$ \\
\hline $\begin{array}{l}\text { Calacarus millingtoniae } \\
\text { Mohanasundaram, } 1982\end{array}$ & · Millingtonia hortensis L.f. & Bignoniaceae & & $\begin{array}{l}\text { Mohanasundaram } 1982 \\
\text { Boczek \& Chandrapatya } \\
\text { 1989b }\end{array}$ \\
\hline $\begin{array}{l}\text { Calacarus mussaendae } \\
\text { Keifer, } 1977\end{array}$ & $\begin{array}{l}\text { Mussaenda philippica } \\
\text { A.bRich. }\end{array}$ & Rubiaceae & - & Keifer $1977 b$ \\
\hline $\begin{array}{l}\text { Calacarus solani } \\
\text { Chandrapatya \& Boczek, } \\
2002\end{array}$ & $\begin{array}{l}\text { Solanum seaforthianum } \\
\text { Andrews }\end{array}$ & Solanaceae & $\begin{array}{l}\text { new } \\
\text { combination }\end{array}$ & $\begin{array}{l}\text { Chandrapatya \& Boczek } \\
2002 \text { b }\end{array}$ \\
\hline $\begin{array}{l}\text { Calepitrimerus michelus } \\
\text { Chandrapatya \& Boczek, } \\
2000\end{array}$ & $\begin{array}{l}\text {-Magnolia champaca (L.) } \\
\text { Baill. ex Pierre }\end{array}$ & Magnoliaceae & - & $\begin{array}{l}\text { Chandrapatya \& Boczek } \\
\text { 2000a }\end{array}$ \\
\hline $\begin{array}{l}\text { Calepitrimerus piperus } \\
\text { Chandrapatya, } 1991\end{array}$ & -Piper sarmentosum Roxb. & Piperaceae & - & $\begin{array}{l}\text { Chandrapatya \& Boczek } \\
\text { 1991b }\end{array}$ \\
\hline $\begin{array}{l}\text { Casearius wormiformis } \\
\text { Boczek \& Chandrapatya, } \\
2001\end{array}$ & - Casearia grewiifolia Vent. & Flacourtiaceae & - & $\begin{array}{l}\text { Boczek \& Chandrapatya } \\
2001\end{array}$ \\
\hline $\begin{array}{l}\text { Catarhinus pennisetosus } \\
\text { Boczek, } 1997\end{array}$ & $\begin{array}{l}\text { Pennisetum purpureum } \\
\text { Schumach. }\end{array}$ & Poaceae & - & $\begin{array}{l}\text { Chandrapatya \& Boczek } \\
1997 b\end{array}$ \\
\hline $\begin{array}{l}\text { Cathetacarus imperatae } \\
\text { (Chandrapatya, 1993) }\end{array}$ & $\begin{array}{l}\text { Imperata cylindrica (L.) } \\
\text { Beauv. }\end{array}$ & Poaceae & $\begin{array}{l}\text { new } \\
\text { combination }\end{array}$ & $\begin{array}{l}\text { Chandrapatya \& Boczek } \\
\text { 1993b }\end{array}$ \\
\hline $\begin{array}{l}\text { Cecidodectes tremae } \\
\text { (Boczek, 1992) }\end{array}$ & $\begin{array}{l}\text { Trema angustifolia } \\
\text { (Planchon) Blume }\end{array}$ & Ulmaceae & $\begin{array}{l}\text { new } \\
\text { combination }\end{array}$ & $\begin{array}{l}\text { Boczek \& Chandrapatya } \\
1992 b\end{array}$ \\
\hline $\begin{array}{l}\text { Cecidophyes ophiopogonis } \\
\text { Keifer, } 1976\end{array}$ & $\begin{array}{l}\text { Ophiopogon japonicus } \\
\text { (Thunb.) Ker.-Gawl. }\end{array}$ & Ruscaceae & emendation & Keifer 1976 \\
\hline $\begin{array}{l}\text { Cecidophyes thailandica } \\
\text { Keifer, } 1975\end{array}$ & ·Ficus microcarpa L.f. & Moraceae & - & Keifer 1975a \\
\hline $\begin{array}{l}\text { Cheiracus sulcatus Keifer, } \\
1977\end{array}$ & - Oryza sativa $\mathrm{L}$. & Poaceae & - & Keifer 1977a \\
\hline $\begin{array}{l}\text { Chiacaphyllisa eugeniae } \\
\text { (Chandrapatya, 1996) }\end{array}$ & -Syzygium cumini (L.) Skeels & Myrtaceae & $\begin{array}{l}\text { new } \\
\text { combination }\end{array}$ & $\begin{array}{l}\text { Boczek \& Chandrapatya } \\
\text { 1996a }\end{array}$ \\
\hline $\begin{array}{l}\text { Chiangmaia diospyrosi } \\
\text { (Chandrapatya \& Boczek, } \\
\text { 2000) }\end{array}$ & Diospyros sp. & Ebenaceae & $\begin{array}{l}\text { new } \\
\text { combination }\end{array}$ & $\begin{array}{l}\text { Chandrapatya \& Boczek } \\
\text { 2000e }\end{array}$ \\
\hline $\begin{array}{l}\text { Chiangmaia longifolii } \\
\text { (Chandrapatya \& Boczek, } \\
\text { 2000) }\end{array}$ & $\begin{array}{l}\text { Polyalthia longifolia (Sonn.) } \\
\text { Thwaites }\end{array}$ & Annonaceae & - & $\begin{array}{l}\text { Chandrapatya \& Boczek } \\
2000 \mathrm{e} \\
\text { Amrine et al. } 2003\end{array}$ \\
\hline $\begin{array}{l}\text { Cisaberoptus kenyae Keifer, } \\
1966\end{array}$ & - Mangifera indica L. & Anacardiaceae & - & Keifer 1966a \\
\hline $\begin{array}{l}\text { Colomerus novahebridensis } \\
\text { Keifer, } 1977\end{array}$ & Cocos nucifera L. & Arecaceae & & $\begin{array}{l}\text { Keifer } 1977 b \\
\text { Chandrapatya et al. } 2014 b\end{array}$ \\
\hline
\end{tabular}




\begin{tabular}{|c|c|c|c|c|}
\hline Eriophyoid mite & Host plant & Host family & $\begin{array}{c}\text { Remarks } \\
\text { onmite }\end{array}$ & Reference for mite \\
\hline $\begin{array}{l}\text { Colopodacus bengalensis } \\
\text { Mohanasundaram, } 1981\end{array}$ & ·Ficus superba (Miq.) & Moraceae & - & $\begin{array}{l}\text { Mohanasundaram } 1981 \\
\text { Chandrapatya \& Boczek } \\
\text { 1993a }\end{array}$ \\
\hline $\begin{array}{l}\text { Combretus thailandus } \\
\text { Boczek \& Chandrapatya, } \\
2001\end{array}$ & $\begin{array}{l}\text { Combretum quadrangulare } \\
\text { Kurz }\end{array}$ & Combretaceae & - & $\begin{array}{l}\text { Boczek \& Chandrapatya } \\
2001\end{array}$ \\
\hline $\begin{array}{l}\text { Coptophylla querci } \\
\text { (Chandrapatya \& Boczek, } \\
\text { 2000) }\end{array}$ & $\begin{array}{l}\text { Quercus mespilifolia Wall. } \\
\text { ex A.DC. }\end{array}$ & Fagaceae & - & $\begin{array}{l}\text { Chandrapatya \& Boczek } \\
2000 f \\
\text { Xue et al. } 2009\end{array}$ \\
\hline $\begin{array}{l}\text { Cosella atalanti (Boczek \& } \\
\text { Chandrapatya, 2000) }\end{array}$ & $\begin{array}{l}\text { Atalantia monophylla L. } \\
\text { (DC.) Corrêa }\end{array}$ & Rutaceae & - & $\begin{array}{l}\text { Boczek \& Chandrapatya } \\
\text { 2000c } \\
\text { Amrine et al. } 2003\end{array}$ \\
\hline Cosella cissi Keifer, 1978 & -Cissus trifoliata (L.) L. & Vitaceae & - & Keifer 1978 \\
\hline $\begin{array}{l}\text { Cosella crotoni (Boczek \& } \\
\text { Chandrapatya, 2000) }\end{array}$ & Croton persimilis Müll. Arg & Euphorbiaceae & - & $\begin{array}{l}\text { Boczek \& Chandrapatya } \\
\text { 2000c } \\
\text { Xue \& Zhang } 2009 \\
\end{array}$ \\
\hline $\begin{array}{l}\text { Cosella erythrini (Boczek \& } \\
\text { Chandrapatya, 2000) }\end{array}$ & Erythrina fusca Lour. & Fabaceae & - & $\begin{array}{l}\text { Boczek \& Chandrapatya } \\
\text { 2000c } \\
\text { Amrine et al. } 2003 \\
\text { Xue \& Zhang } 2009 \\
\end{array}$ \\
\hline $\begin{array}{l}\text { Cosella trangii } \\
\text { Chandrapatya, } \\
\text { Konvipasruang \& Amrine, } \\
2016\end{array}$ & $\begin{array}{l}\text { Dimocarpus longan Lour. } \\
\text { ssp. longan var. longan }\end{array}$ & Sapindaceae & $\begin{array}{l}\text { new } \\
\text { combination, } \\
\text { replacement } \\
\text { name }\end{array}$ & $\begin{array}{l}\text { Boczek \& Chandrapatya } \\
2000 \text { c }\end{array}$ \\
\hline $\begin{array}{l}\text { Criocarpus tropicalis } \\
\text { Boczek \& Chandrapatya, } \\
2000\end{array}$ & $\begin{array}{l}\text { Artocarpus lacucha Buch.- } \\
\text { Ham. }\end{array}$ & Moraceae & - & $\begin{array}{l}\text { Boczek \& Chandrapatya } \\
\text { 2000e }\end{array}$ \\
\hline $\begin{array}{l}\text { Diptilomiopus aglaiae } \\
\text { (Chandrapatya \& Boczek, } \\
\text { 2002) }\end{array}$ & Aglaia $\mathrm{sp}$ & Meliaceae & - & $\begin{array}{l}\text { Chandrapatya \& Boczek } \\
\text { 2002a } \\
\text { Cramer et al. } 2005 \\
\end{array}$ \\
\hline $\begin{array}{l}\text { Diptilomiopus } \\
\text { anthocephaliae } \\
\text { (Chandrapatya \& Boczek, } \\
\text { 2002) }\end{array}$ & $\begin{array}{l}\text {-Neolamarckia cadamba } \\
\text { (Roxb.) Bosser }\end{array}$ & Rubiaceae & - & $\begin{array}{l}\text { Chandrapatya \& Boczek } \\
\text { 2002a } \\
\text { Cramer et al. } 2005\end{array}$ \\
\hline $\begin{array}{l}\text { Diptilomiopus artabotrysi } \\
\text { (Boczek, 1991) }\end{array}$ & $\begin{array}{l}\text { Artabotrys hexapetalus (L.f) } \\
\text { Bhandari }\end{array}$ & Annonaceae & - & $\begin{array}{l}\text { Chandrapatya \& Boczek } \\
\text { 1991b } \\
\text { Hong \& Zhang } 1997\end{array}$ \\
\hline $\begin{array}{l}\text { Diptilomiopus azadirachtae } \\
\text { (Boczek, 1992) }\end{array}$ & $\begin{array}{l}\text {-Azadirachta indica A. Juss. } \\
\text { var. siamensis Valeton }\end{array}$ & Meliaceae & - & $\begin{array}{l}\text { Boczek \& Chandrapatya } \\
\text { 1992c } \\
\text { Hong \& Zhang } 1997 \\
\end{array}$ \\
\hline $\begin{array}{l}\text { Diptilomiopus barringtoniae } \\
\text { (Chandrapatya, 1992) }\end{array}$ & $\begin{array}{l}\text { Barringtonia acutangula } \\
\text { (L.) Gaertn.subsp. } \\
\text { acutangula }\end{array}$ & Lecythidaceae & - & $\begin{array}{l}\text { Boczek \& Chandrapatya } \\
\text { 1992c } \\
\text { Hong \& Zhang } 1997 \\
\end{array}$ \\
\hline $\begin{array}{l}\text { Diptilomiopus benjaminae } \\
\text { (Boczek \& Chandrapatya, } \\
\text { 2002) }\end{array}$ & Ficus benjamina $\mathrm{L}$. & Moraceae & - & $\begin{array}{l}\text { Boczek \& Chandrapatya } \\
2002 \\
\text { Cramer et al. } 2005\end{array}$ \\
\hline $\begin{array}{l}\text { Diptilomiopus boueae } \\
\text { (Chandrapatya \& Boczek, } \\
\text { 2002) }\end{array}$ & - Bouea macrophylla Griff. & Anacardiaceae & - & $\begin{array}{l}\text { Chandrapatya \& Boczek } \\
\text { 2002a } \\
\text { Cramer et al. } 2005\end{array}$ \\
\hline $\begin{array}{l}\text { Diptilomiopus cerberae } \\
\text { (Chandrapatya, 1998) }\end{array}$ & Cerbera odollam Gaertn & Apocynaceae & - & $\begin{array}{l}\text { Boczek \& Chandrapatya } \\
1998 \text { b } \\
\text { Cramer et al. } 2005\end{array}$ \\
\hline $\begin{array}{l}\text { Diptilomiopus coreiae } \\
\text { (Chandrapatya \& Boczek, } \\
\text { 2002) }\end{array}$ & -Morinda coreia Buch.-Ham. & Rubiaceae & - & $\begin{array}{l}\text { Chandrapatya \& Boczek } \\
2002 \text { b } \\
\text { Cramer et al. } 2005\end{array}$ \\
\hline
\end{tabular}




\begin{tabular}{|c|c|c|c|c|}
\hline Eriophyoid mite & Host plant & Host family & $\begin{array}{c}\text { Remarks } \\
\text { onmite }\end{array}$ & Reference for mite \\
\hline $\begin{array}{l}\text { Diptilomiopus cythereae } \\
\text { (Chandrapatya, 1991) }\end{array}$ & $\begin{array}{l}\text { Spondias dulcis Sol. ex } \\
\text { Parkinson }\end{array}$ & Anacardiaceae & - & $\begin{array}{l}\text { Chandrapatya \& Boczek } \\
\text { 1991a } \\
\text { Hong \& Zhang } 1997\end{array}$ \\
\hline $\begin{array}{l}\text { Diptilomiopus elaeocarpi } \\
\text { (Boczek, 1991) }\end{array}$ & $\begin{array}{l}\text { Elaeocarpus hygrophilus } \\
\text { Kurz }\end{array}$ & Elaeocarpaceae & - & $\begin{array}{l}\text { Chandrapatya \& Boczek } \\
\text { 1991a } \\
\text { Hong \& Zhang } 1997\end{array}$ \\
\hline $\begin{array}{l}\text { Diptilomiopus eucalypti } \\
\text { (Boczek, 1991) }\end{array}$ & $\begin{array}{l}\text { Eucalyptus camaldulensis } \\
\text { Dehnh. }\end{array}$ & Myrtaceae & - & $\begin{array}{l}\text { Chandrapatya \& Boczek } \\
\text { 1991b } \\
\text { Hong \& Zhang } 1997\end{array}$ \\
\hline $\begin{array}{l}\text { Diptilomiopus jasminiae } \\
\text { (Chandrapatya \& Boczek, } \\
\text { 2001) }\end{array}$ & Jasminum auriculatum Vahl & Oleaceae & - & $\begin{array}{l}\text { Chandrapatya \& Boczek } \\
\text { 2001a } \\
\text { Cramer et al. } 2005\end{array}$ \\
\hline $\begin{array}{l}\text { Diptilomiopus khatkhaii } \\
\text { Konvipasruang \& } \\
\text { Chandrapatya, } 2015\end{array}$ & Oxyceros horridus Lour. & Rubiaceae & $\begin{array}{l}\text { new genus, } \\
\text { new species }\end{array}$ & $\begin{array}{l}\text { Konvipasruang } \\
\text { \&Chandrapatya, } 2015\end{array}$ \\
\hline $\begin{array}{l}\text { Diptilomiopus knorri Keifer, } \\
1974\end{array}$ & Gardenia sp. & Rubiaceae & - & Keifer 1974 \\
\hline $\begin{array}{l}\text { Diptilomiopus languasi } \\
\text { (Boczek, 1991) }\end{array}$ & -Alpinia galanga (L.) Willd. & Zingiberaceae & - & $\begin{array}{l}\text { Chandrapatya \& Boczek } \\
\text { 1991b } \\
\text { Hong \& Zhang } 1997\end{array}$ \\
\hline $\begin{array}{l}\text { Diptilomiopus mandongii } \\
\text { Konvipasruang \& } \\
\text { Chandrapatya, } 2015\end{array}$ & - Cordia dichotoma G. Forst. & Boraginaceae & $\begin{array}{l}\text { new genus, } \\
\text { new species }\end{array}$ & $\begin{array}{l}\text { Konvipasruang \& } \\
\text { Chandrapatya, } 2015\end{array}$ \\
\hline $\begin{array}{l}\text { Diptilomiopus melastomae } \\
\text { (Boczek \& Chandrapatya, } \\
\text { 2002) }\end{array}$ & $\begin{array}{l}\text {-Melastoma malabathricum } \\
\text { L. ssp. normale (D.Don) } \\
\text { F. K. Meyer }\end{array}$ & Melastomaceae & - & $\begin{array}{l}\text { Boczek \& Chandrapatya } \\
2002 \\
\text { Cramer et al. } 2005\end{array}$ \\
\hline $\begin{array}{l}\text { Diptilomiopus meliae } \\
\text { (Boczek, 1998) }\end{array}$ & - Melia azedarach $\mathrm{L}$. & Meliaceae & - & $\begin{array}{l}\text { Boczek \& Chandrapatya } \\
1998 b \\
\text { Cramer et al. } 2005\end{array}$ \\
\hline $\begin{array}{l}\text { Diptilomiopus morindae } \\
\text { (Boczek, 1998) }\end{array}$ & -Morinda citrifolia $\mathrm{L}$. & Rubiaceae & - & $\begin{array}{l}\text { Boczek \& Chandrapatya } \\
1998 b \\
\text { Cramer et al. } 2005\end{array}$ \\
\hline $\begin{array}{l}\text { Diptilomiopus musae } \\
\text { (Chandrapatya, 1998) }\end{array}$ & - Musa acuminata Colla & Musaceae & - & $\begin{array}{l}\text { Chandrapatya \& Boczek } \\
1998 \\
\text { Cramer et al. } 2005\end{array}$ \\
\hline $\begin{array}{l}\text { Diptilomiopus pamithus } \\
\text { (Boczek \& Chandrapatya, } \\
\text { 1989) }\end{array}$ & -Mangifera indica $\mathrm{L}$. & Anacardiaceae & emendation & $\begin{array}{l}\text { Boczek \& Chandrapatya } \\
\text { 1989a } \\
\text { Chandrapatya \& Boczek, } \\
\text { 1991a } \\
\text { Hong \& Zhang } 1997\end{array}$ \\
\hline $\begin{array}{l}\text { Diptilomiopus racemosae } \\
\text { (Chandrapatya \& Boczek, } \\
\text { 2001) }\end{array}$ & Ficus racemosa $\mathrm{L}$. & Moraceae & - & $\begin{array}{l}\text { Chandrapatya \& Boczek } \\
\text { 2001a } \\
\text { Cramer et al. } 2005\end{array}$ \\
\hline $\begin{array}{l}\text { Diptilomiopus riciniae } \\
\text { (Boczek \& Chandrapatya, } \\
\text { 2002) }\end{array}$ & - Ricinus communis $\mathrm{L}$. & Euphorbiaceae & - & $\begin{array}{l}\text { Boczek \& Chandrapatya } \\
2002 \\
\text { Cramer et al. } 2005\end{array}$ \\
\hline $\begin{array}{l}\text { Diptilomiopus } \\
\text { sakaena Chandrapatya, } \\
\text { Konvipasruang \& Amrine, } \\
2016\end{array}$ & $\begin{array}{l}\text { Combretum quadrangulare } \\
\text { Kurz }\end{array}$ & Combretaceae & $\begin{array}{l}\text { replacement } \\
\text { name }\end{array}$ & $\begin{array}{l}\text { Chandrapatya \& Boczek } \\
\text { 2002a }\end{array}$ \\
\hline $\begin{array}{l}\text { Diptilomiopus sandorici } \\
\text { (Chandrapatya, 1991) }\end{array}$ & $\begin{array}{l}\text {-Sandoricum koetjape (Burm. } \\
\text { f.) Merr. }\end{array}$ & Meliaceae & - & $\begin{array}{l}\text { Chandrapatya \& Boczek } \\
\text { 1991a } \\
\text { Hong \& Zhang } 1997\end{array}$ \\
\hline $\begin{array}{l}\text { Diptilomiopus securinegus } \\
\text { Boczek, } 1992\end{array}$ & $\begin{array}{l}\text { Flueggea leucopyrus } \\
\text { (Willd.) }\end{array}$ & $\begin{array}{l}\text { K. D.Koenig ex } \\
\text { Roxb. }\end{array}$ & $\begin{array}{l}\text { Euphorbia- } \\
\text { ceae }\end{array}$ & $\begin{array}{l}\text { Boczek \& Chandrapatya } \\
\text { 1992a } \\
\text { Hong \& Zhang } 1997\end{array}$ \\
\hline
\end{tabular}




\begin{tabular}{|c|c|c|c|c|}
\hline Eriophyoid mite & Host plant & Host family & $\begin{array}{l}\text { Remarks } \\
\text { onmite }\end{array}$ & Reference for mite \\
\hline $\begin{array}{l}\text { Diptilomiopus strebli } \\
\text { (Boczek, 1992) }\end{array}$ & -Streblus asper Lour. & Moraceae & - & $\begin{array}{l}\text { Boczek \& Chandrapatya } \\
\text { 1992c } \\
\text { Hong \& Zhang } 1997\end{array}$ \\
\hline $\begin{array}{l}\text { Diptilomiopus swieteniae } \\
\text { (Chandrapatya, 1998) }\end{array}$ & -Swietenia macrophylla King & Meliaceae & - & $\begin{array}{l}\text { Chandrapatya \& Boczek } \\
1998 \\
\text { Cramer et al. } 2005\end{array}$ \\
\hline $\begin{array}{l}\text { Diptilomiopus thaianae } \\
\text { (Boczek, 1991) }\end{array}$ & $\begin{array}{l}\text { Syzygium malaccense L. } \\
\text { Merr. \& L. M. Perry }\end{array}$ & Myrtaceae & - & $\begin{array}{l}\text { Chandrapatya \& Boczek } \\
\text { 1991a } \\
\text { Hong \& Zhang } 1997\end{array}$ \\
\hline $\begin{array}{l}\text { Diptilomiopus thunbergiae } \\
\text { (Boczek \& Chandrapatya, } \\
\text { 2002) }\end{array}$ & · Thunbergia laurifolia Lindl. & Acanthaceae & - & $\begin{array}{l}\text { Boczek \& Chandrapatya } \\
2002 \\
\text { Cramer et al. } 2005\end{array}$ \\
\hline $\begin{array}{l}\text { Diptiloplatus megagrastis } \\
\text { Keifer, } 1975\end{array}$ & $\begin{array}{l}\text { Gigantochloa ligulata } \\
\text { Gamble }\end{array}$ & $\begin{array}{l}\text { Poaceae, } \\
\text { Bambuseae }\end{array}$ & - & Keifer 1975a \\
\hline $\begin{array}{l}\text { Duabangus chiangmai } \\
\text { Chandrapatya \& Boczek, } \\
2000\end{array}$ & $\begin{array}{l}\text { Duabanga grandiflora } \\
\text { (Roxb. ex DC.) Walp. }\end{array}$ & Sonneratiaceae & - & $\begin{array}{l}\text { Chandrapatya \& Boczek } \\
\text { 2000d }\end{array}$ \\
\hline $\begin{array}{l}\text { Echinacrus psophocarpi } \\
\text { (Chandrapatya, 1992) }\end{array}$ & $\begin{array}{l}\text { Psophocarpus } \\
\text { tetragonolobus (L.) DC. }\end{array}$ & Fabaceae & - & $\begin{array}{l}\text { Boczek \& Chandrapatya } \\
\text { 1992c } \\
\text { Ozdikmen } 2008\end{array}$ \\
\hline $\begin{array}{l}\text { Ekaphyes anacardi Boczek } \\
\& \text { Chandrapatya, } 2000\end{array}$ & - Anacardium occidentale L. & Anacardiaceae & - & $\begin{array}{l}\text { Boczek \& Chandrapatya } \\
\text { 2000f }\end{array}$ \\
\hline $\begin{array}{l}\text { Epitrimerus acanthi } \\
\text { (Chandrapatya, 1992) }\end{array}$ & -Acanthus ebracteatus Vahl & Acanthaceae & $\begin{array}{l}\text { new } \\
\text { combination }\end{array}$ & $\begin{array}{l}\text { Boczek \& Chandrapatya } \\
\text { 1992b }\end{array}$ \\
\hline $\begin{array}{l}\text { Epitrimerus altissimus } \\
\text { Chandrapatya \& Boczek, } \\
2001\end{array}$ & Ficus altissima Blume & Moraceae & - & $\begin{array}{l}\text { Chandrapatya \& Boczek } \\
\text { 2001b }\end{array}$ \\
\hline $\begin{array}{l}\text { Epitrimerus cannae Boczek } \\
1998\end{array}$ & - Canna indica $\mathrm{L}$. & Cannaceae & - & $\begin{array}{l}\text { Chandrapatya \& Boczek } \\
1998\end{array}$ \\
\hline $\begin{array}{l}\text { Epitrimerus combreti } \\
\text { Boczek \& Chandrapatya, } \\
2001\end{array}$ & $\begin{array}{l}\text { Combretum quadrangulare } \\
\text { Kurz }\end{array}$ & Combretaceae & - & $\begin{array}{l}\text { Boczek \& Chandrapatya } \\
2001\end{array}$ \\
\hline $\begin{array}{l}\text { Epitrimerus ellipticus } \\
\text { Boczek \& Chandrapatya, } \\
2000\end{array}$ & -Morinda coreia Buch.-Ham. & Rubiaceae & - & $\begin{array}{l}\text { Boczek \& Chandrapatya } \\
\text { 2000e }\end{array}$ \\
\hline $\begin{array}{l}\text { Epitrimerus tinosporus } \\
\text { Chandrapatya \& Boczek, } \\
2000\end{array}$ & $\begin{array}{l}\text {-Tinospora crispa (L.) Hook. } \\
\text { f. \& Thomson }\end{array}$ & Menispermaceae & - & $\begin{array}{l}\text { Chandrapatya \& Boczek } \\
\text { 2000a }\end{array}$ \\
\hline $\begin{array}{l}\text { Epitrimerus zingiberi } \\
\text { Boczek, } 1998\end{array}$ & -Zingiber officinale Roscoe & Zingiberaceae & - & $\begin{array}{l}\text { Chandrapatya \& Boczek } \\
1998\end{array}$ \\
\hline $\begin{array}{l}\text { Eriophyes manilkarae } \\
\text { (Keifer, 1977) }\end{array}$ & $\begin{array}{l}\text {-Manilkara hexandra (Roxb.) } \\
\text { Dubard }\end{array}$ & Sapotaceae & - & $\begin{array}{l}\text { Keifer 1977a } \\
\text { Amrine \& Stasny } 1994\end{array}$ \\
\hline $\begin{array}{l}\text { Floracarus perrepae } \\
\text { Knihinicki \& Boczek, } 2002\end{array}$ & $\begin{array}{l}\text { Lygodium microphyllum } \\
\text { (Cav.) R.Br. }\end{array}$ & Schizaeaceae & - & Knihinicki \& Boczek 2002 \\
\hline $\begin{array}{l}\text { Garcinyes madannis Boczek } \\
\& \text { Chandrapatya, } 2000\end{array}$ & $\begin{array}{l}\text { Garcinia schomburgkiana } \\
\text { Pierre }\end{array}$ & Clusiaceae & - & $\begin{array}{l}\text { Boczek \& Chandrapatya } \\
2000 f\end{array}$ \\
\hline $\begin{array}{l}\text { Garcinyes mangosteenis } \\
\text { Boczek \& Chandrapatya, } \\
2000\end{array}$ & - Garcinia mangostana $\mathrm{L}$. & Clusiaceae & - & $\begin{array}{l}\text { Boczek \& Chandrapatya } \\
2000 f\end{array}$ \\
\hline $\begin{array}{l}\text { Hainanarus heliciopsus } \\
\text { (Chandrapatya \& Boczek, } \\
\text { 2000) }\end{array}$ & $\begin{array}{l}\text { ·Heliciopsis terminalis } \\
\text { (Kurz) Sleumer }\end{array}$ & Proteaceae & $\begin{array}{l}\text { new } \\
\text { combination }\end{array}$ & $\begin{array}{l}\text { Chandrapatya \& Boczek } \\
\text { 2000d }\end{array}$ \\
\hline $\begin{array}{l}\text { Hoderus roseus (Keifer, } \\
\text { 1975) }\end{array}$ & $\begin{array}{l}\text {-Lepisanthes rubiginosa } \\
\text { (Roxb.) Leenth. }\end{array}$ & Sapindaceae & - & $\begin{array}{l}\text { Keifer 1975b } \\
\text { Manson \& Gerson } 1996\end{array}$ \\
\hline
\end{tabular}




\begin{tabular}{|c|c|c|c|c|}
\hline Eriophyoid mite & Host plant & Host family & $\begin{array}{c}\text { Remarks } \\
\text { onmite }\end{array}$ & Reference for mite \\
\hline $\begin{array}{l}\text { Jaranasia anamensiae } \\
\text { Chandrapatya \& Boczek, } \\
2000\end{array}$ & Parinari anamensis Hance & $\begin{array}{l}\text { Chrysobalana- } \\
\text { ceae }\end{array}$ & - & $\begin{array}{l}\text { Chandrapatya \& Boczek } \\
2000 \text { b }\end{array}$ \\
\hline $\begin{array}{l}\text { Jutarus benjaminae Boczek } \\
\& \text { Chandrapatya, } 1989\end{array}$ & Ficus benjamina L. & Moraceae & - & $\begin{array}{l}\text { Boczek \& Chandrapatya } \\
\text { 1989a }\end{array}$ \\
\hline $\begin{array}{l}\text { Kaella flacourtiae } \\
\text { (Chandrapatya \& Boczek, } \\
\text { 2002) }\end{array}$ & Flacourtia sp. & Flacourtiaceae & - & $\begin{array}{l}\text { Chandrapatya \& Boczek } \\
2002 \text { b } \\
\text { Amrine et al. } 2003\end{array}$ \\
\hline $\begin{array}{l}\text { Khamroka tomentosi } \\
\text { Konvipasruang } \\
\text { \&Chandrapatya, } 2015 \\
\end{array}$ & $\begin{array}{l}\text { Ellipanthus tomentosus } \\
\text { Kurz }\end{array}$ & Connaraceae & $\begin{array}{l}\text { new genus, } \\
\text { new species }\end{array}$ & $\begin{array}{l}\text { Konvipasruang \& } \\
\text { Chandrapatya, } 2015\end{array}$ \\
\hline $\begin{array}{l}\text { Khanthongella suregadi } \\
\text { (Boczek \& Chandrapatya, } \\
2000 \text { ) }\end{array}$ & $\begin{array}{l}\text { Suregada multiflora } \\
\text { (A. Juss.) Baill. }\end{array}$ & Euphorbiaceae & $\begin{array}{l}\text { new genus, } \\
\text { new } \\
\text { combination }\end{array}$ & $\begin{array}{l}\text { Boczek \& Chandrapatya } \\
\text { 2000a } \\
\text { Chandrapatya et al. } 2015\end{array}$ \\
\hline $\begin{array}{l}\text { Knorella blumeanae Xue \& } \\
\text { Zhang, } 2009\end{array}$ & $\begin{array}{l}\text { Bambusa sp. } \\
\text {-Bambusa blumeana Schult. } \\
\text { \& Schult.f. } \\
\text {-Bambusa multiplex (Lour.) } \\
\text { Raeusch. ex Schult. \& } \\
\text { Schult.f. }\end{array}$ & $\begin{array}{l}\text { Poaceae, } \\
\text { Bambuseae }\end{array}$ & 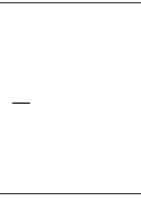 & $\begin{array}{l}\text { Boczek \& Chandrapatya } \\
\text { 1998a } \\
\text { Xue \& Zhang } 2009 \\
\text { Konvipasruang et al. 2012a }\end{array}$ \\
\hline $\begin{array}{l}\text { Knorella gigantochloae } \\
\text { Keifer, } 1975\end{array}$ & $\begin{array}{l}\text { Gigantochloa ligulata } \\
\text { Gamble }\end{array}$ & $\begin{array}{l}\text { Poaceae, } \\
\text { Bambuseae }\end{array}$ & - & Keifer 1975a \\
\hline $\begin{array}{l}\text { Knorella thailandica Hong, } \\
1996\end{array}$ & $\begin{array}{l}\text { ·Bambusa sp. } \\
\text { ·Bambusa vulgaris Schrad. } \\
\text { ex J.C.Wendl. }\end{array}$ & $\begin{array}{l}\text { Poaceae, } \\
\text { Bambuseae }\end{array}$ & - & $\begin{array}{l}\text { Chandrapatya \& Boczek } \\
\text { 1993b } \\
\text { Hong } 1996\end{array}$ \\
\hline $\begin{array}{l}\text { Kolacarus bambusae } \\
\text { Boczek, } 1998\end{array}$ & $\begin{array}{l}\text {-Bambusa sp. } \\
\text {-Bambusa bambos (L.) Voss } \\
\text {-Bambusa blumeana } \text { Schult. } \\
\text { \& Schult.f. } \\
\text {-Bambusa multiplex (Lour.) } \\
\text { Raeusch. ex Schult. \& } \\
\text { Schult.f. } \\
\text {-Bambusa ventricosa } \\
\text { McClure } \\
\text {-Bambusa vulgaris Schrad. } \\
\text { ex J. C.Wendl. } \\
\text {-Dendrocalamus latiflorus } \\
\text { Munro }\end{array}$ & $\begin{array}{l}\text { Poaceae, } \\
\text { Bambuseae }\end{array}$ & - & $\begin{array}{l}\text { Boczek \& Chandrapatya } \\
\text { 1998a } \\
\text { Konvipasruang et al. 2012a }\end{array}$ \\
\hline $\begin{array}{l}\text { Kosacarus dipterocarpi } \\
\text { (Chandrapatya \& Boczek, } \\
\text { 2000) }\end{array}$ & $\begin{array}{l}\text { Dipterocarpus alatus Roxb. } \\
\text { ex G. Don }\end{array}$ & Dipterocarpaceae & - & $\begin{array}{l}\text { Chandrapatya \& Boczek } \\
\text { 2000c } \\
\text { Chandrapatya et al. 2014a }\end{array}$ \\
\hline $\begin{array}{l}\text { Kosacarus rayongi } \\
\text { Chandrapatya, } \\
\text { Konvipasruang \& Amrine, } \\
2014\end{array}$ & $\begin{array}{l}\text { Dipterocarpus alatus Roxb. } \\
\text { ex G.Don }\end{array}$ & Dipterocarpaceae & - & Chandrapatya et al. 2014a \\
\hline $\begin{array}{l}\text { Kradukarus suregadi } \\
\text { Chandrapatya, } \\
\text { Konvipasruang \& Amrine, } \\
2016\end{array}$ & $\begin{array}{l}\text { Suregada multiflora } \\
\text { (A.Juss.) Baill. }\end{array}$ & Euphorbiaceae & $\begin{array}{l}\text { new genus, } \\
\text { new species }\end{array}$ & Chandrapatya et al. 2016a \\
\hline $\begin{array}{l}\text { Lamyacarus longani } \\
\text { Chandrapatya, } \\
\text { Konvipasruang \& Amrine, } \\
2016\end{array}$ & $\begin{array}{l}\text { Dimocarpus longan Lour. } \\
\text { ssp. longan var. longan }\end{array}$ & Sapindaceae & $\begin{array}{l}\text { new genus, } \\
\text { new species }\end{array}$ & Chandrapatya et al. 2016a \\
\hline $\begin{array}{l}\text { Leipothrix bangkokus } \\
\text { (Chandrapatya, 1996) }\end{array}$ & Cyperus sp. & Cyperaceae & - & $\begin{array}{l}\text { Boczek \& Chandrapatya } \\
1996 a \\
\text { Chetverikov } 2005\end{array}$ \\
\hline
\end{tabular}




\begin{tabular}{|c|c|c|c|c|}
\hline Eriophyoid mite & Host plant & Host family & $\begin{array}{c}\text { Remarks } \\
\text { onmite }\end{array}$ & Reference for mite \\
\hline $\begin{array}{l}\text { Levonga caseariae } \\
\text { (Chandrapatya, 1997) }\end{array}$ & - Casearia grewiifolia Vent. & Flacourtiaceae & - & $\begin{array}{l}\text { Chandrapatya \& Boczek } \\
\text { 1997b } \\
\text { Amrine et al. } 2003\end{array}$ \\
\hline $\begin{array}{l}\text { Lipacarus engeniae (Boczek, } \\
\text { 1992) }\end{array}$ & $\begin{array}{l}\text { Syzygium samarangense } \\
\text { (Blume) Merrill \& L. M. } \\
\text { Perry }\end{array}$ & Myrtaceae & $\begin{array}{l}\text { new genus, } \\
\text { new } \\
\text { combination }\end{array}$ & $\begin{array}{l}\text { Boczek \& Chandrapatya } \\
\text { 1992b } \\
\text { Chandrapatya et al. 2016b }\end{array}$ \\
\hline $\begin{array}{l}\text { Lithocarus thomsoni } \\
\text { Chandrapatya \& Boczek, } \\
2000\end{array}$ & $\begin{array}{l}\text {-Lithocarpus thomsonii } \\
\text { (Miq.) Rehder }\end{array}$ & Fagaceae & - & $\begin{array}{l}\text { Chandrapatya \& Boczek } \\
\text { 2000e }\end{array}$ \\
\hline $\begin{array}{l}\text { Longanella lamyai } \\
\text { Chandrapatya, } \\
\text { Konvipasruang \& Amrine, } \\
2016\end{array}$ & $\begin{array}{l}\text { - Dimocarpus longan Lour. } \\
\text { ssp. longan var. longan }\end{array}$ & Sapindaceae & $\begin{array}{l}\text { new genus, } \\
\text { new species }\end{array}$ & Chandrapatya et al. 2016a \\
\hline $\begin{array}{l}\text { Makruta citri (Boczek, } \\
\text { 1996) }\end{array}$ & Citrus hystrix DC. & Rutaceae & $\begin{array}{l}\text { new genus, } \\
\text { new } \\
\text { combination }\end{array}$ & $\begin{array}{l}\text { Boczek \& Chandrapatya } \\
\text { 1996a } \\
\text { Chandrapatya et al. } 2015\end{array}$ \\
\hline $\begin{array}{l}\text { Mangophyes siami } \\
\text { Chandrapatya \& Boczek, } \\
2001\end{array}$ & - Mangifera indica $\mathrm{L}$. & Anacardiaceae & - & $\begin{array}{l}\text { Chandrapatya \& Boczek } \\
2001 \text { b }\end{array}$ \\
\hline $\begin{array}{l}\text { Metaculus mangiferae } \\
\text { (Attiah, 1955) }\end{array}$ & - Mangifera indica $\mathrm{L}$. & Anacardiaceae & & $\begin{array}{l}\text { Attiah } 1955 \\
\text { ChannaBasavanna } 1966 \\
\text { Chandrapatya \& Boczek } \\
\text { 1991c }\end{array}$ \\
\hline $\begin{array}{l}\text { Mimenacarus glutinosae } \\
\text { (Boczek, 1996) }\end{array}$ & $\begin{array}{l}\text {-Litsea glutinosa (Lour.) } \\
\text { C. B. Robinson }\end{array}$ & Lauraceae & $\begin{array}{l}\text { new genus, } \\
\text { new } \\
\text { combination }\end{array}$ & $\begin{array}{l}\text { Chandrapatya \& Boczek } \\
1996 \\
\text { Chandrapatya et al. } 2015\end{array}$ \\
\hline $\begin{array}{l}\text { Neocatarhinus namtai } \\
\text { Konvipasruang, } \\
\text { Chandrapatya \& Amrine, } \\
2012\end{array}$ & $\begin{array}{l}\cdot \text { Bambusa sp. } \\
\text {-Bambusa ventricosa } \\
\text { McClure } \\
\cdot \text { Bambusa vulgaris Schrad. } \\
\text { ex J.C.Wendl. } \\
\text {-Dendrocalamus asper } \\
\text { (Schult. \& Schult.f.) Backer } \\
\text { ex K.Heyne } \\
\text {-Melocanna humilis Roep. ex } \\
\text { Trin. }\end{array}$ & $\begin{array}{l}\text { Poaceae, } \\
\text { Bambuseae }\end{array}$ & - & $\begin{array}{l}\text { Boczek \& Chandrapatya } \\
\text { 1998a } \\
\text { Konvipasruang et al. 2012a }\end{array}$ \\
\hline $\begin{array}{l}\text { Neometaculus alpini } \\
\text { (Chandrapatya \& Boczek, } \\
\text { 2000) }\end{array}$ & $\begin{array}{l}\text { Alpinia zerumbet (Pers.) } \\
\text { B. L.Burtt \& R. M. Sm. }\end{array}$ & Zingiberaceae & $\begin{array}{l}\text { new } \\
\text { combination }\end{array}$ & $\begin{array}{l}\text { Chandrapatya \& Boczek } \\
\text { 2000f }\end{array}$ \\
\hline $\begin{array}{l}\text { Neometaculus terminaliae } \\
\text { (Boczek \& Chandrapatya, } \\
\text { 1989) }\end{array}$ & - Terminalia catappa $\mathrm{L}$. & Combretaceae & - & $\begin{array}{l}\text { Boczek \& Chandrapatya } \\
\text { 1989a } \\
\text { Maimala et al. } 2002\end{array}$ \\
\hline $\begin{array}{l}\text { Neometaculus trewi } \\
\text { (Chandrapatya \& Boczek, } \\
\text { 2000) }\end{array}$ & - Trewia nudiflora $\mathrm{L}$. & Euphorbiaceae & $\begin{array}{l}\text { new } \\
\text { combination }\end{array}$ & $\begin{array}{l}\text { Chandrapatya \& Boczek } \\
\text { 2000f }\end{array}$ \\
\hline $\begin{array}{l}\text { Neserella capreifoliae Meyer } \\
\& \text { Ueckermann, } 1989\end{array}$ & ·Ficus hispida L. f. & Moraceae & & $\begin{array}{l}\text { Meyer \& Ueckermann } \\
1989 \\
\text { Chandrapatya \& Boczek } \\
\text { 2000d } \\
\text { Amrine et al. } 2003\end{array}$ \\
\hline $\begin{array}{l}\text { Nonthaburinus litchi } \\
\text { Chandrapatya, } 1996\end{array}$ & - Litchi chinensis Sonn. & Sapindaceae & - & $\begin{array}{l}\text { Chandrapatya \& Boczek } \\
1996\end{array}$ \\
\hline Notallus nerii Keifer, 1975 & - Nerium oleander L. & Apocynaceae & - & Keifer $1975 \mathrm{a}$ \\
\hline
\end{tabular}




\begin{tabular}{|c|c|c|c|c|}
\hline Eriophyoid mite & Host plant & Host family & $\begin{array}{c}\text { Remarks } \\
\text { onmite }\end{array}$ & Reference for mite \\
\hline $\begin{array}{l}\text { Paniculatus microcosus } \\
\text { Boczek \& Chandrapatya, } \\
2000\end{array}$ & - Microcos paniculata L. & Tiliaceae & - & $\begin{array}{l}\text { Boczek \& Chandrapatya } \\
2000 \text { b }\end{array}$ \\
\hline $\begin{array}{l}\text { Paniculatus samoengus } \\
\text { Boczek \& Chandrapatya, } \\
2000\end{array}$ & - Microcos paniculata L. & Tiliaceae & - & $\begin{array}{l}\text { Boczek \& Chandrapatya } \\
2000 \text { b }\end{array}$ \\
\hline $\begin{array}{l}\text { Paraphytoptus erythrinae } \\
\text { Keifer, } 1975\end{array}$ & Erythrina sp. & Fabaceae & - & Keifer $1975 b$ \\
\hline $\begin{array}{l}\text { Parinarus anamensus } \\
\text { Chandrapatya \& Boczek, } \\
2000\end{array}$ & Parinari anamensis Hance & $\begin{array}{l}\text { Chrysobalana- } \\
\text { ceae }\end{array}$ & - & $\begin{array}{l}\text { Chandrapatya \& Boczek } \\
\text { 2000b }\end{array}$ \\
\hline $\begin{array}{l}\text { Pentacecidophyes litchi } \\
\text { (Boczek, 1996) }\end{array}$ & -Litchi chinensis Sonn. & Sapindaceae & $\begin{array}{l}\text { new } \\
\text { combination }\end{array}$ & $\begin{array}{l}\text { Chandrapatya \& Boczek } \\
1996 \\
\text { Chandrapatya et al } 2016 \text { b }\end{array}$ \\
\hline $\begin{array}{l}\text { Petanovicia cerberae } \\
\text { Boczek, } 1996\end{array}$ & - Cerbera odollam Gaertn & Apocynaceae & - & $\begin{array}{l}\text { Boczek \& Chandrapatya } \\
1996 \mathrm{~b}\end{array}$ \\
\hline $\begin{array}{l}\text { Phaicus bambusae } \\
\text { (ChannaBassavanna, 1966) }\end{array}$ & $\begin{array}{l}\cdot \text { Bambusa sp. } \\
\text {-Bambusa ventricosa } \\
\text { McClure }\end{array}$ & $\begin{array}{l}\text { Poaceae, } \\
\text { Bambuseae }\end{array}$ & - & $\begin{array}{l}\text { Boczek \& Chandrapatya } \\
\text { 1998a } \\
\text { Konvipasruang et al. 2012a }\end{array}$ \\
\hline $\begin{array}{l}\text { Phaicus panticis (Keifer, } \\
\text { 1997) }\end{array}$ & $\begin{array}{l}\text { Bambusa ventricosa } \\
\text { McClure }\end{array}$ & $\begin{array}{l}\text { Poaceae, } \\
\text { Bambuseae }\end{array}$ & - & $\begin{array}{l}\text { Keifer } 1977 b \\
\text { Konvipasruang et al. 2012a }\end{array}$ \\
\hline $\begin{array}{l}\text { Phayomoca talutus } \\
\text { (Chandrapatya \& Boczek, } \\
\text { 2000) }\end{array}$ & -Shorea roxburghii G.Don & Dipterocarpaceae & $\begin{array}{l}\text { new genus, } \\
\text { new } \\
\text { combination }\end{array}$ & $\begin{array}{l}\text { Chandrapatya \& Boczek } \\
\text { 2000d } \\
\text { Chandrapatya et al. } 2015\end{array}$ \\
\hline $\begin{array}{l}\text { Phutsona ervatamiae } \\
\text { (Chandrapatya, 1991) }\end{array}$ & · Gardenia jasminoides J.Ellis & Rubiaceae & $\begin{array}{l}\text { new genus, } \\
\text { new } \\
\text { combination }\end{array}$ & $\begin{array}{l}\text { Chandrapatya \& Boczek } \\
\text { 1991a } \\
\text { Chandrapatya et al 2016b }\end{array}$ \\
\hline $\begin{array}{l}\text { Phyllocoptes azimae Boczek, } \\
1991\end{array}$ & $\begin{array}{l}\text {-Azima sarmentosa (Blume) } \\
\text { Benth. \& Hook.f. }\end{array}$ & Salvadoraceae & - & $\begin{array}{l}\text { Chandrapatya \& Boczek } \\
\text { 1991c }\end{array}$ \\
\hline $\begin{array}{l}\text { Phyllocoptes breyniae } \\
\text { Boczek \& Chandrapatya, } \\
1989\end{array}$ & $\begin{array}{l}\text { Breynia vitis-idaea (Burm.f.) } \\
\text { C. E. C.Fisch. }\end{array}$ & Euphorbiaceae & - & $\begin{array}{l}\text { Boczek \& Chandrapatya } \\
1989 \text { b }\end{array}$ \\
\hline $\begin{array}{l}\text { Phyllocoptes ficifolii Boczek } \\
\text { \& Chandrapatya, } 2000\end{array}$ & Ficus hispida L.f. & Moraceae & - & $\begin{array}{l}\text { Boczek \& Chandrapatya } \\
\text { 2000d }\end{array}$ \\
\hline $\begin{array}{l}\text { Phyllocoptes indicae Keifer, } \\
1975\end{array}$ & $\begin{array}{l}\text { Flacourtia indica (Burm.f.) } \\
\text { Merr. }\end{array}$ & Flacourtiaceae & - & Keifer 1975a \\
\hline $\begin{array}{l}\text { Phyllocoptes takhoppae } \\
\text { Chandrapatya, } \\
\text { Konvipasruang, \& Amrine, } \\
2016\end{array}$ & $\begin{array}{l}\text { Flacourtia indica (Burm.f.) } \\
\text { Merr. }\end{array}$ & Flacourtiaceae & $\begin{array}{l}\text { new } \\
\text { combination, } \\
\text { replacement } \\
\text { name }\end{array}$ & $\begin{array}{l}\text { Boczek \& Chandrapatya } \\
\text { 2000c }\end{array}$ \\
\hline $\begin{array}{l}\text { Phyllocoptruta azadirachtae } \\
\text { (Chandrapatya, 1992) }\end{array}$ & $\begin{array}{l}\text { Azadirachta indica A.Juss. } \\
\text { var. siamensis Valeton }\end{array}$ & Meliaceae & $\begin{array}{l}\text { new } \\
\text { combination }\end{array}$ & $\begin{array}{l}\text { Boczek \& Chandrapatya } \\
1992 b\end{array}$ \\
\hline $\begin{array}{l}\text { Phyllocoptruta maerimae } \\
\text { Boczek \& Chandrapatya, } \\
2000\end{array}$ & - Bischofia javanica Blume & Phyllanthaceae & - & $\begin{array}{l}\text { Boczek \& Chandrapatya } \\
\text { 2000d }\end{array}$ \\
\hline $\begin{array}{l}\text { Phyllocoptruta malligai } \\
\text { Mohanasundaram, } 1980\end{array}$ & $\begin{array}{l}\text { Ipomoea staphylina Roem. } \\
\text { \& Schult. }\end{array}$ & Convolvulaceae & - & $\begin{array}{l}\text { Mohanasundaram } 1980 \\
\text { Chandrapatya \& Boczek } \\
\text { 1993a }\end{array}$ \\
\hline $\begin{array}{l}\text { Phyllocoptruta meliae } \\
\text { Keifer, } 1977\end{array}$ & -Melia sp. & Meliaceae & - & Keifer 1977a \\
\hline $\begin{array}{l}\text { Phyllocoptruta morindae } \\
\text { Keifer, } 1977\end{array}$ & $\cdot$ Morinda citrifolia $\mathrm{L}$. & Rubiaceae & - & Keifer $1977 b$ \\
\hline $\begin{array}{l}\text { Phyllocoptruta musae } \\
\text { Keifer, } 1955\end{array}$ & 'Musa' paradisiaca $\mathrm{L}$. & Musaceae & & Keifer, 1955 \\
\hline
\end{tabular}




\begin{tabular}{|c|c|c|c|c|}
\hline Eriophyoid mite & Host plant & Host family & $\begin{array}{c}\text { Remarks } \\
\text { onmite }\end{array}$ & Reference for mite \\
\hline $\begin{array}{l}\text { Phyllocoptruta oleivora } \\
\text { (Ashmead, 1879) }\end{array}$ & $\begin{array}{l}\text { Citrus sp. } \\
\text {-Citrus aurantiifolia } \\
\text { (Christm.) Swingle } \\
\text {-Citrus maxima (Burm.) } \\
\text { Merr. } \\
\text {-Citrus reticulata } \text { Blanco } \\
\text {-Citrus sinensis (L.) Osbeck } \\
\text { - Fortunella japonica } \\
\text { (Thunb.) Swingle }\end{array}$ & Rutaceae & - & Ashmead 1879 \\
\hline $\begin{array}{l}\text { Protumescoptes sandorici } \\
\text { (Chandrapatya \& Boczek, } \\
2000)\end{array}$ & $\begin{array}{l}\text { Sandoricum koetjape (Burm. } \\
\text { f.) Merr. }\end{array}$ & Meliaceae & $\begin{array}{l}\text { new } \\
\text { combination }\end{array}$ & $\begin{array}{l}\text { Chandrapatya \& Boczek } \\
\text { 2000c }\end{array}$ \\
\hline $\begin{array}{l}\text { Quadriporca indicae } \\
\text { (Boczek, 1997) }\end{array}$ & - Mangifera indica $\mathrm{L}$. & Anacardiaceae & - & $\begin{array}{l}\text { Chandrapatya \& Boczek } \\
\text { 1997a } \\
\text { Chandrapatya \& Boczek } \\
\text { 2002b } \\
\text { Xue \& Zhang } 2009\end{array}$ \\
\hline $\begin{array}{l}\text { Randius oppositifolii Boczek } \\
\text { \& Chandrapatya, } 2000\end{array}$ & $\begin{array}{l}\text {-Aidia densiflora (Wall.) } \\
\text { Masam. }\end{array}$ & Rubiaceae & - & $\begin{array}{l}\text { Boczek \& Chandrapatya } \\
\text { 2000a }\end{array}$ \\
\hline $\begin{array}{l}\text { Ranongus eugenus } \\
\text { Chandrapatya \& Boczek, } \\
2000\end{array}$ & $\begin{array}{l}\text { Syzygium samarangense } \\
\text { (Blume) Merrill \& L. M. } \\
\text { Perry }\end{array}$ & Myrtaceae & - & $\begin{array}{l}\text { Chandrapatya \& Boczek } \\
\text { 2000c }\end{array}$ \\
\hline $\begin{array}{l}\text { Rapinarus maphoki } \\
\text { Chandrapatya, } \\
\text { Konvipasruang \& Amrine, } \\
2016\end{array}$ & -Parinari anamensis Hance & $\begin{array}{l}\text { Chrysobalana- } \\
\text { ceae }\end{array}$ & $\begin{array}{l}\text { new genus, } \\
\text { new species }\end{array}$ & Chandrapatya et al 2016a \\
\hline $\begin{array}{l}\text { Rhombacus eucalyptifoliae } \\
\text { Boczek, } 1995\end{array}$ & $\begin{array}{l}\text { Eucalyptus camaldulensis } \\
\text { Dehnh. }\end{array}$ & Myrtaceae & - & $\begin{array}{l}\text { Chandrapatya \& Boczek } \\
\text { 1991c } \\
\text { Boczek \& Petanovic } 1995\end{array}$ \\
\hline $\begin{array}{l}\text { Salaya takhronami } \\
\text { Konvipasruang \& } \\
\text { Chandrapatya, 2015 } \\
\end{array}$ & $\begin{array}{l}\text {-Sisyrolepis muricata (Pierre) } \\
\text { Leenh. }\end{array}$ & Sapindaceae & $\begin{array}{l}\text { new genus, } \\
\text { new species }\end{array}$ & $\begin{array}{l}\text { Konvipasruang \& } \\
\text { Chandrapatya, } 2015\end{array}$ \\
\hline $\begin{array}{l}\text { Shevtchenkella arfeuilleae } \\
\text { Chandrapatya \& Boczek, } \\
2000\end{array}$ & $\begin{array}{l}\text { Arfeuillea arborescens } \\
\text { Pierre }\end{array}$ & Sapindaceae & emendation & $\begin{array}{l}\text { Chandrapatya \& Boczek } \\
\text { 2000a }\end{array}$ \\
\hline $\begin{array}{l}\text { Siamina cuminae Boczek, } \\
1993\end{array}$ & -Syzygium cumini (L.) Skeels & Myrtaceae & - & $\begin{array}{l}\text { Chandrapatya \& Boczek } \\
\text { 1993a }\end{array}$ \\
\hline $\begin{array}{l}\text { Siracharus dipterocarpi } \\
\text { Chandrapatya \& Boczek, } \\
2000\end{array}$ & $\begin{array}{l}\text { Dipterocarpus alatus Roxb. } \\
\text { ex G. Don }\end{array}$ & Dipterocarpaceae & - & $\begin{array}{l}\text { Chandrapatya \& Boczek } \\
\text { 2000c }\end{array}$ \\
\hline $\begin{array}{l}\text { Spinacus pagonis Keifer, } \\
1979\end{array}$ & $\cdot$ Mangifera indica $\mathrm{L}$. & Anacardiaceae & - & $\begin{array}{l}\text { Keifer } 1979 \\
\text { Chandrapatya \& Boczek } \\
\text { 1991c }\end{array}$ \\
\hline $\begin{array}{l}\text { Steopa bauhiniae } \\
\text { (Chandrapatya \& Boczek, } \\
\text { 2001) }\end{array}$ & -Bauhinia purpurea $\mathrm{L}$. & Fabaceae & - & $\begin{array}{l}\text { Chandrapatya \& Boczek } \\
\text { 2001c } \\
\text { Amrine et al. } 2003\end{array}$ \\
\hline $\begin{array}{l}\text { Surapoda artabotrysi } \\
\text { Boczek \& Chandrapatya, } \\
1989\end{array}$ & $\begin{array}{l}\text { Artabotrys hexapetalus (L.f) } \\
\text { Bhandari }\end{array}$ & Annonaceae & - & $\begin{array}{l}\text { Boczek \& Chandrapatya } \\
1989 \text { b }\end{array}$ \\
\hline $\begin{array}{l}\text { Suthamus chiangmi } \\
\text { Chandrapatya \& Boczek, } \\
2000\end{array}$ & -Parinari anamensis Hance & $\begin{array}{l}\text { Chrysobalana- } \\
\text { ceae }\end{array}$ & & $\begin{array}{l}\text { Chandrapatya \& Boczek } \\
\text { 2000b }\end{array}$ \\
\hline $\begin{array}{l}\text { Tegolophus artocarpi Keifer, } \\
1977\end{array}$ & $\begin{array}{l}\text { Artocarpus heterophyllus } \\
\text { Lam. }\end{array}$ & Moraceae & - & Keifer $1977 b$ \\
\hline $\begin{array}{l}\text { Tegolophus cassii (Boczek } \\
\text { \& Chandrapatya, 2000) }\end{array}$ & $\begin{array}{l}\text { Senna siamea (Lam.) H. S. } \\
\text { Irwin \& Barneby }\end{array}$ & Fabaceae & $\begin{array}{l}\text { new } \\
\text { combination }\end{array}$ & $\begin{array}{l}\text { Boczek \& Chandrapatya } \\
\text { 2000a }\end{array}$ \\
\hline
\end{tabular}




\begin{tabular}{|c|c|c|c|c|}
\hline Eriophyoid mite & Host plant & Host family & $\begin{array}{c}\text { Remarks } \\
\text { onmite }\end{array}$ & Reference for mite \\
\hline $\begin{array}{l}\text { Tegonotus convolvuli } \\
\text { (Channabasavanna, 1966) }\end{array}$ & -Ipomoea aquatica Forssk. & Convolvulaceae & - & $\begin{array}{l}\text { Channabasavanna, } 1966 \\
\text { Jeppson \& Keifer } 1975\end{array}$ \\
\hline $\begin{array}{l}\text { Tegonotus streblusi Boczek, } \\
1997\end{array}$ & Streblus asper Lour. & Moraceae & emendation & $\begin{array}{l}\text { Chandrapatya \& Boczek } \\
\text { 1997a }\end{array}$ \\
\hline $\begin{array}{l}\text { Tegophyes benjamini } \\
\text { Chandrapatya \& Boczek, } \\
2001\end{array}$ & Ficus benjamina L. & Moraceae & - & $\begin{array}{l}\text { Chandrapatya \& Boczek } \\
2001 \text { b }\end{array}$ \\
\hline $\begin{array}{l}\text { Tegophyes chonburi } \\
\text { Chandrapatya \& Boczek, } \\
2001\end{array}$ & Ficus sp. & Moraceae & - & $\begin{array}{l}\text { Chandrapatya \& Boczek } \\
2001 \text { b }\end{array}$ \\
\hline $\begin{array}{l}\text { Tetra albiziae Chandrapatya } \\
\text { \& Boczek, } 2001\end{array}$ & ·Albizia lebbeck (L.) Benth. & Fabaceae & - & $\begin{array}{l}\text { Chandrapatya \& Boczek } \\
\text { 2001a }\end{array}$ \\
\hline $\begin{array}{l}\text { Tetra caseariae Boczek, } \\
1997\end{array}$ & Casearia grewiifolia Vent. & Flacourtiaceae & - & $\begin{array}{l}\text { Chandrapatya \& Boczek } \\
\text { 1997a }\end{array}$ \\
\hline $\begin{array}{l}\text { Tetra cassiae Das \& } \\
\text { Chakrabarti, } 1994\end{array}$ & - Cassia fistula L. & Fabaceae & - & $\begin{array}{l}\text { Chandrapatya \& Boczek } \\
\text { 1997a } \\
\text { Das \& Chakrabarti } 1994\end{array}$ \\
\hline $\begin{array}{l}\text { Tetra centrosemae } \\
\text { Chandrapatya \& Boczek, } \\
2000\end{array}$ & $\begin{array}{l}\text { Centrosema pubescens } \\
\text { Benth. }\end{array}$ & Fabaceae & - & $\begin{array}{l}\text { Chandrapatya \& Boczek } \\
2000 \mathrm{~g}\end{array}$ \\
\hline $\begin{array}{l}\text { Tetra diospyrosae } \\
\text { Chandrapatya, } 1993\end{array}$ & -Diospyros rhodocalyx Kurz & Ebenaceae & - & $\begin{array}{l}\text { Chandrapatya \& Boczek } \\
\text { 1993a }\end{array}$ \\
\hline $\begin{array}{l}\text { Tetra fistulae Chandrapatya } \\
\text { \& Boczek, } 2000\end{array}$ & - Cassia fistula L. & Fabaceae & - & $\begin{array}{l}\text { Chandrapatya \& Boczek } \\
2000 \mathrm{~g}\end{array}$ \\
\hline $\begin{array}{l}\text { Tetra lepisanthae Boczek \& } \\
\text { Chandrapatya, } 2000\end{array}$ & $\begin{array}{l}\text {-Lepisanthes rubiginosa } \\
\text { (Roxb.) Leenth. }\end{array}$ & Sapindaceae & - & $\begin{array}{l}\text { Boczek \& Chandrapatya } \\
2000 f\end{array}$ \\
\hline $\begin{array}{l}\text { Tetra minuta (Chandrapatya } \\
\& \text { Boczek, 2000) }\end{array}$ & - Bauhinia variegata $\mathrm{L}$. & Fabaceae & emendation & $\begin{array}{l}\text { Chandrapatya \& Boczek } \\
2000 f\end{array}$ \\
\hline $\begin{array}{l}\text { Tetra salaya Chandrapatya } \\
\text { \& Boczek, } 2001\end{array}$ & $\begin{array}{l}\text { Feroniella lucida (Scheff.) } \\
\text { Swingle }\end{array}$ & Rutaceae & - & $\begin{array}{l}\text { Chandrapatya \& Boczek } \\
\text { 2001a }\end{array}$ \\
\hline $\begin{array}{l}\text { Tetra samaniae } \\
\text { Chandrapatya \& Boczek, } \\
2000\end{array}$ & $\begin{array}{l}\text { Samanea saman (Jacq.) } \\
\text { Meer. }\end{array}$ & Fabaceae & - & $\begin{array}{l}\text { Chandrapatya \& Boczek } \\
2000 \mathrm{~g}\end{array}$ \\
\hline $\begin{array}{l}\text { Tetra stipularisis Xue \& } \\
\text { Zhang, } 2009\end{array}$ & $\begin{array}{l}\text {-Bridelia stipularis (L.) } \\
\text { Blume }\end{array}$ & Euphorbiaceae & - & $\begin{array}{l}\text { Chandrapatya \& Boczek } \\
2000 \mathrm{~g} \\
\text { Xue \& Zhang } 2009\end{array}$ \\
\hline $\begin{array}{l}\text { Thacra piperasia Keifer, } \\
1978\end{array}$ & $\begin{array}{l}\text {-Piper nigrum L. } \\
\text { ·Piper sarmentosum Roxb. }\end{array}$ & Piperaceae & - & Keifer 1978 \\
\hline $\begin{array}{l}\text { Thailandus diospyrosae } \\
\text { Chandrapatya, } 1997\end{array}$ & -Diospyros gracilis Fletch. & Ebenaceae & - & $\begin{array}{l}\text { Chandrapatya \& Boczek } \\
\text { 1997b }\end{array}$ \\
\hline $\begin{array}{l}\text { Vareeboona mangiferae } \\
\text { (Chandrapatya, 1997) }\end{array}$ & - Mangifera indica $\mathrm{L}$. & Anacardiaceae & $\begin{array}{l}\text { new genus, } \\
\text { new } \\
\text { combination }\end{array}$ & $\begin{array}{l}\text { Chandrapatya \& Boczek } \\
\text { 1997a } \\
\text { Chandrapatya et al. } 2015\end{array}$ \\
\hline $\begin{array}{l}\text { Vasates leucopyri } \\
\text { Chandrapatya, } 1992\end{array}$ & $\begin{array}{l}\text { Flueggea leucopyrus } \\
\text { (Willd.) K. D. Koenig ex } \\
\text { Roxb. }\end{array}$ & Euphorbiaceae & - & $\begin{array}{l}\text { Boczek \& Chandrapatya } \\
\text { 1992a }\end{array}$ \\
\hline $\begin{array}{l}\text { Vasates pavetis Ghosh, } \\
\text { Mondal \& Chakrabarti, } 1986\end{array}$ & -Morinda citrifolia L. & Rubiaceae & - & $\begin{array}{l}\text { Ghosh, Mondal \& } \\
\text { Chakrabarti } 1986 \\
\text { Chandrapatya \& Boczek } \\
\text { 1993a }\end{array}$ \\
\hline $\begin{array}{l}\text { Vasates scaevolai } \\
\text { Chandrapatya, } 1996\end{array}$ & $\begin{array}{l}\text {-Scaevola taccada (Gaertn.) } \\
\text { Roxb. }\end{array}$ & Goodeniaceae & - & $\begin{array}{l}\text { Boczek \& Chandrapatya } \\
1996 \mathrm{~b}\end{array}$ \\
\hline $\begin{array}{l}\text { Vasates spinosae } \\
\text { Chandrapatya, } 1996\end{array}$ & Citharexylum spinosum L. & Verbenaceae & - & $\begin{array}{l}\text { Boczek \& Chandrapatya } \\
1996 b\end{array}$ \\
\hline
\end{tabular}




\begin{tabular}{|c|c|c|c|c|}
\hline Eriophyoid mite & Host plant & Host family & $\begin{array}{c}\text { Remarks } \\
\text { onmite }\end{array}$ & Reference for mite \\
\hline $\begin{array}{l}\text { Vasates spondiasi Boczek \& } \\
\text { Chandrapatya, } 1989\end{array}$ & $\begin{array}{l}\text { Spondias bipinnata Airy- } \\
\text { Shaw \& Forman }\end{array}$ & Anacardiaceae & - & $\begin{array}{l}\text { Boczek \& Chandrapatya } \\
\text { 1989a }\end{array}$ \\
\hline $\begin{array}{l}\text { Vimola cassiae } \\
\text { Chandrapatya \& Boczek, } \\
2001\end{array}$ & $\begin{array}{l}\text { Senna siamea }(\text { Lam.) H. S. } \\
\text { Irwin \& Barneby }\end{array}$ & Fabaceae & - & $\begin{array}{l}\text { Chandrapatya \& Boczek } \\
2001 \text { c }\end{array}$ \\
\hline $\begin{array}{l}\text { Vimola psidiae } \\
\text { Chandrapatya, } 1996 \\
\end{array}$ & -Psidium guajava $\mathrm{L}$. & Myrtaceae & - & $\begin{array}{l}\text { Boczek \& Chandrapatya } \\
\text { 1996a }\end{array}$ \\
\hline $\begin{array}{l}\text { Vimola quisqualii } \\
\text { (Chandrapatya, 1997) }\end{array}$ & $\begin{array}{l}\text { Combretum indicum (L.) } \\
\text { DeFilipps }\end{array}$ & Combretaceae & $\begin{array}{l}\text { new } \\
\text { combination }\end{array}$ & $\begin{array}{l}\text { Chandrapatya \& Boczek } \\
\text { 1997b }\end{array}$ \\
\hline Vimola syzygii Boczek, 1992 & $\begin{array}{l}\text { Syzygium cumini (L.) Skeels } \\
\text {-Syzygium samarangense } \\
\text { (Blume) Merrill \& L. M. } \\
\text { Perry }\end{array}$ & Myrtaceae & - & $\begin{array}{l}\text { Boczek \& Chandrapatya } \\
\text { 1992a }\end{array}$ \\
\hline $\begin{array}{l}\text { Vimola terminalis } \\
\text { (Chandrapatya \& Boczek, } \\
\text { 2000) }\end{array}$ & - Terminalia catappa $\mathrm{L}$. & Combretaceae & $\begin{array}{l}\text { new } \\
\text { combination }\end{array}$ & $\begin{array}{l}\text { Chandrapatya \& Boczek } \\
\text { 2000b }\end{array}$ \\
\hline $\begin{array}{l}\text { Visinus dimocarpi } \\
\text { Chandrapatya, } 1996 \\
\end{array}$ & $\begin{array}{l}\text { Dimocarpus longan Lour. } \\
\text { ssp. longan var. longan }\end{array}$ & Sapindaceae & - & $\begin{array}{l}\text { Chandrapatya \& Boczek } \\
1996\end{array}$ \\
\hline $\begin{array}{l}\text { Wangthonga phayomae } \\
\text { Chandrapatya, } \\
\text { Konvipasruang \& Amrine, } \\
2016 .\end{array}$ & -Shorea roxburghii G.Don & Dipterocarpaceae & $\begin{array}{l}\text { new genus, } \\
\text { new species }\end{array}$ & Chandrapatya et al 2016a \\
\hline $\begin{array}{l}\text { Wanleelagus cerberus } \\
\text { Boczek, } 1997\end{array}$ & Cerbera odollam Gaertn & Apocynaceae & emendation & $\begin{array}{l}\text { Chandrapatya \& Boczek } \\
1997 \text { b }\end{array}$ \\
\hline $\begin{array}{l}\text { Yangnarus dipterocarpi } \\
\text { Chandrapatya, } \\
\text { Konvipasruang \& Amrine, } \\
2016\end{array}$ & $\begin{array}{l}\text { - Dipterocarpus alatus Roxb. } \\
\text { ex G.Don }\end{array}$ & Dipterocarpaceae & $\begin{array}{l}\text { new genus, } \\
\text { new species }\end{array}$ & Chandrapatya et al 2016a \\
\hline
\end{tabular}

lychee, Aceria longana Boczek \& Knihinicki on longan, Aceria mangiferae Sayed on mango, and Phyllocoptruta oleivora (Ashmead) on citrus. The majority of eriophyoid mites in Thailand are considered vagrant, causing no visible damage, although some species-such as those in the genus Calacarus - cause rust on leaves but do not affect yield.

From our examination of the taxonomic characters, we concluded that the scientific names of 50 mites previously identified needed to be justified. Changes made included moving to different genera when a new combination of names was assigned (22 species); assignment of new combination and replacement names ( 2 species); assignment of a replacement name (2 species); emendation of the name ( 7 species); and placement as junior synonym ( 9 species) (Table 2$)$, as well as assignment of a new name at the generic level (8 species) (Table 3 ). In addition, we identified 8 new genera and 10 new species for those mites in the collection that had not yet been given a scientific name (Table 4). In this paper, we provide a new name, Diptilomiopus sakaena Chandrapatya, Konvipasruang \& Amrine, 2016 for Vilaia combretae Chandrapatya \& Boczek, 2002, then Diptilomiopus combretae (Chandrapatya \& Boczek, 2002), junior homonym of Diptilomiopus combretae Wei \& Lu, 2001. The species epithet is after the common name of the host plant, Sakaena. Aculops chonburi Chandrapatya, Konvipasruang \& Amrine, 2016 for Aculops desmodii Chandrapatya \& Boczek, 2000 since Aculops desmodii Chandrapatya \& 
Table 2. List of new combinations, replacement names, emendations, and junior synonyms for 42 previously described eriophyoid species in Thailand

\begin{tabular}{|c|c|c|c|}
\hline New assignment & Original assignment & $\begin{array}{l}\text { Reference for } \\
\text { original assignment }\end{array}$ & Reasons for moving to new genus \\
\hline \multicolumn{4}{|c|}{ New combinations } \\
\hline \multicolumn{4}{|c|}{ Eriophyidae, Phyllocoptinae, Acaricalini } \\
\hline $\begin{array}{l}\text { Chiacaphyllisa eugeniae } \\
\text { (Chandrapatya, 1996) }\end{array}$ & $\begin{array}{l}\text { Acaphylla eugeniae } \\
\text { Chandrapatya, } 1996\end{array}$ & $\begin{array}{l}\text { Boczek \& } \\
\text { Chandrapatya 1996a }\end{array}$ & Antaxial genual seta $l$ " on leg II present. \\
\hline $\begin{array}{l}\text { Protumescoptes sandorici } \\
\text { (Chandrapatya \& Boczek, } \\
2000)\end{array}$ & $\begin{array}{l}\text { Tumescoptes sandorici } \\
\text { Chandrapatya \& } \\
\text { Boczek, } 2000\end{array}$ & $\begin{array}{l}\text { Chandrapatya \& } \\
\text { Boczek 2000c }\end{array}$ & $\begin{array}{l}\text { Dorsal annuli fused immediately behind } \\
\text { prodorsal shield, forming an "extended } \\
\text { shield." Tibial, genu II, and opisthosomal } \\
\text { setae } d \text { and } e \text { absent. }\end{array}$ \\
\hline \multicolumn{4}{|c|}{ Eriophyidae, Phyllocoptinae, Calacarini } \\
\hline $\begin{array}{l}\text { Calacarus cerberi } \\
\text { (Chandrapatya \& Boczek, } \\
\text { 2001) }\end{array}$ & $\begin{array}{l}\text { Callyntrotus cerberi } \\
\text { Chandrapatya \& } \\
\text { Boczek, } 2001\end{array}$ & $\begin{array}{l}\text { Chandrapatya \& } \\
\text { Boczek 2001c }\end{array}$ & $\begin{array}{l}\text { Was moved from Phyllocoptini to Calacarini } \\
\text { because scapular setae } s c \text { absent, scapular } \\
\text { tubercles present. Opisthosoma with five } \\
\text { ridges. }\end{array}$ \\
\hline $\begin{array}{l}\text { Calacarus duranti } \\
\text { (Chandrapatya \& Boczek, } \\
\text { 2001) }\end{array}$ & $\begin{array}{l}\text { Callyntrotus duranti } \\
\text { Chandrapatya \& } \\
\text { Boczek, } 2001\end{array}$ & $\begin{array}{l}\text { Chandrapatya \& } \\
\text { Boczek 2001c }\end{array}$ & $\begin{array}{l}\text { Was moved from Phyllocoptini to Calacarini } \\
\text { because scapular setae } s c \text { absent, scapular } \\
\text { tubercles present. Opisthosoma with five } \\
\text { ridges. }\end{array}$ \\
\hline $\begin{array}{l}\text { Calacarus solani } \\
\text { (Chandrapatya \& Boczek, } \\
\text { 2002) }\end{array}$ & $\begin{array}{l}\text { Callyntrotus solani } \\
\text { Chandrapatya \& } \\
\text { Boczek, } 2002\end{array}$ & $\begin{array}{l}\text { Chandrapatya \& } \\
\text { Boczek 2002b }\end{array}$ & $\begin{array}{l}\text { Was moved from Phyllocoptini to Calacarini } \\
\text { because scapular setae } s c \text { absent, scapular } \\
\text { tubercles present.Opisthosoma with five } \\
\text { ridges. }\end{array}$ \\
\hline \multicolumn{4}{|c|}{ Eriophyidae, Phyllocoptinae, Phyllocoptini } \\
\hline $\begin{array}{l}\text { Bischofius anthocephali } \\
\text { (Boczek \& Chandrapatya, } \\
\text { 2000) }\end{array}$ & $\begin{array}{l}\text { Tegonotus } \\
\text { anthocephali Boczek } \\
\text { \& Chandrapatya, } 2000\end{array}$ & $\begin{array}{l}\text { Boczek \& } \\
\text { Chandrapatya 2000d }\end{array}$ & $\begin{array}{l}\text { Was moved from Tegonotini to Phyllocopini } \\
\text { because lateral lobe absent. }\end{array}$ \\
\hline $\begin{array}{l}\text { Cecidodectes tremae (Boczek, } \\
\text { 1992) }\end{array}$ & $\begin{array}{l}\text { Criophyes tremae } \\
\text { Boczek, } 1992\end{array}$ & $\begin{array}{l}\text { Boczek \& } \\
\text { Chandrapatya 1992b }\end{array}$ & $\begin{array}{l}\text { Was moved from Anthocoptini to } \\
\text { Phyllocoptini owing to position of scapular } \\
\text { setae } s c \text {. Differed from Cecidodectes euzonus } \\
\text { Nalepa by having } 4 \text { rays on empodium (not } \\
5 \text { rays) and being found on different host } \\
\text { species. }\end{array}$ \\
\hline $\begin{array}{l}\text { Epitrimerus acanthi } \\
\text { (Chandrapatya, 1992) }\end{array}$ & $\begin{array}{l}\text { Acalox acanthi } \\
\text { Chandrapatya, } 1992\end{array}$ & $\begin{array}{l}\text { Boczek \& } \\
\text { Chandrapatya } 1992 \text { b }\end{array}$ & $\begin{array}{l}\text { Has three very distinct dorsal ridges; median } \\
\text { ridge fairly short and ending in a trough; } \\
\text { ridges are broad and flat on the upper } \\
\text { surface. }\end{array}$ \\
\hline $\begin{array}{l}\text { Hainanarus heliciopsus } \\
\text { (Chandrapatya \& Boczek, } \\
\text { 2000) }\end{array}$ & $\begin{array}{l}\text { Calepitrimerus } \\
\text { heliciopsus } \\
\text { Chandrapatya \& } \\
\text { Boczek, } 2000\end{array}$ & $\begin{array}{l}\text { Chandrapatya \& } \\
\text { Boczek 2000d }\end{array}$ & $\begin{array}{l}\text { First dorsal annulus broad, equal three to } \\
\text { four annuli; antaxial genual seta } l \text { " on leg II } \\
\text { absent. }\end{array}$ \\
\hline $\begin{array}{l}\text {-Neometaculus alpini } \\
\text { (Chandrapatya \& Boczek, } \\
\text { 2000) }\end{array}$ & $\begin{array}{l}\text { Epitrimerus alpini } \\
\text { Chandrapatya \& } \\
\text { Boczek, } 2000\end{array}$ & $\begin{array}{l}\text { Chandrapatya \& } \\
\text { Boczek 2000f }\end{array}$ & Anterior seta $1 b$ on coxisternum I absent. \\
\hline $\begin{array}{l}\text {-Neometaculus trewi } \\
\text { (Chandrapatya \& Boczek, } \\
\text { 2000) }\end{array}$ & $\begin{array}{l}\text { Epitrimerus trewi } \\
\text { Chandrapatya \& } \\
\text { Boczek, } 2000\end{array}$ & $\begin{array}{l}\text { Chandrapatya \& } \\
\text { Boczek 2000f }\end{array}$ & Anterior seta $1 b$ on coxisternum I absent. \\
\hline $\begin{array}{l}\text { Pentacecidophyes litchi } \\
\text { (Boczek, 1996) }\end{array}$ & $\begin{array}{l}\text { Abacarus litchi } \\
\text { Boczek, } 1996\end{array}$ & $\begin{array}{l}\text { Chandrapatya \& } \\
\text { Boczek } 1996\end{array}$ & $\begin{array}{l}\text { Was moved from Colomerini to } \\
\text { Phyllocoptini because too much space } \\
\text { between the coxisterna of leg II and the } \\
\text { genital shield; genitalia are not appressed to } \\
\text { coxae; longitudinal ridges on coverflap are } \\
\text { not arranged in two ranks. }\end{array}$ \\
\hline $\begin{array}{l}\text { Phyllocoptruta azadirachtae } \\
\text { (Chandrapatya, 1992) }\end{array}$ & $\begin{array}{l}\text { Phyllocoptes } \\
\text { azadirachtae } \\
\text { Chandrapatya, } 1992\end{array}$ & $\begin{array}{l}\text { Boczek \& } \\
\text { Chandrapatya 1992b }\end{array}$ & Dorsal opisthosoma with a wide furrow. \\
\hline
\end{tabular}




\begin{tabular}{|c|c|c|c|}
\hline New assignment & Original assignment & $\begin{array}{l}\text { Reference for } \\
\text { original assignment }\end{array}$ & Reasons for moving to new genus \\
\hline \multicolumn{4}{|c|}{ Eriophyidae, Phyllocoptinae, Anthocoptini } \\
\hline $\begin{array}{l}\text { Aculops albizi (Boczek \& } \\
\text { Chandrapatya, 2000) }\end{array}$ & $\begin{array}{l}\text { Gammaphytoptus } \\
\text { albizi Boczek \& } \\
\text { Chandrapatya, } 2000\end{array}$ & $\begin{array}{l}\text { Boczek \& } \\
\text { Chandrapatya 2000e }\end{array}$ & $\begin{array}{l}\text { Was moved from Cecidophyni to } \\
\text { Anthocoptini because too much space } \\
\text { between coxisterna of leg II and the genital } \\
\text { shield; genitalia are not appressed to coxae. }\end{array}$ \\
\hline $\begin{array}{l}\text { Aculops cannae (Boczek, } \\
\text { 1992) }\end{array}$ & $\begin{array}{l}\text { Vasates cannae } \\
\text { Boczek, } 1992\end{array}$ & $\begin{array}{l}\text { Boczek \& } \\
\text { Chandrapatya 1992b }\end{array}$ & $\begin{array}{l}\text { Was moved from Phyllocoptini to } \\
\text { Anthocoptini because of scapular setae } s c \\
\text { situated on rear shield margin. }\end{array}$ \\
\hline $\begin{array}{l}\text { Aculus morindai } \\
\text { (Chandrapatya, 1996) }\end{array}$ & $\begin{array}{l}\text { Vasates morindai } \\
\text { Chandrapatya, } 1996\end{array}$ & $\begin{array}{l}\text { Boczek \& } \\
\text { Chandrapatya 1996b }\end{array}$ & $\begin{array}{l}\text { Was moved from Phyllocoptini to } \\
\text { Anthocoptini because of scapular setae } s c \\
\text { situated on rear shield margin. }\end{array}$ \\
\hline $\begin{array}{l}\text { Aculus tetraspermae } \\
\text { (Chandrapatya, 1992) }\end{array}$ & $\begin{array}{l}\text { Criotacus tetraspermae } \\
\text { Chandrapatya, } 1992\end{array}$ & $\begin{array}{l}\text { Boczek \& } \\
\text { Chandrapatya 1992a }\end{array}$ & $\begin{array}{l}\text { Was moved from Phyllocoptini to } \\
\text { Anthocoptini because of scapular setae } s c \\
\text { situated on rear shield margin. }\end{array}$ \\
\hline $\begin{array}{l}\text {-Tegolophus cassii (Boczek \& } \\
\text { Chandrapatya, 2000) }\end{array}$ & $\begin{array}{l}\text { Tegoprionus } \\
\text { cassii Boczek \& } \\
\text { Chandrapatya, } 2000\end{array}$ & $\begin{array}{l}\text { Boczek \& } \\
\text { Chandrapatya 2000a }\end{array}$ & Dorsal opisthosoma with three ridges. \\
\hline \multicolumn{4}{|c|}{ Diptilomiopidae, Diptilomiopinae } \\
\hline $\begin{array}{l}\text { Chiangmaia diospyrosi } \\
\text { (Chandrapatya \& Boczek, } \\
\text { 2000) }\end{array}$ & $\begin{array}{l}\text { Diptacus diospyrosi } \\
\text { Chandrapatya \& } \\
\text { Boczek, } 2000\end{array}$ & $\begin{array}{l}\text { Chandrapatya \& } \\
\text { Boczek 2000e }\end{array}$ & Paraxial tibial seta $l$ ' absent. \\
\hline $\begin{array}{l}\cdot \text { Vimola quisqualii } \\
\text { (Chandrapatya, 1997) }\end{array}$ & $\begin{array}{l}\text { Rhynacus quisqualii } \\
\text { Chandrapatya, } 1997\end{array}$ & $\begin{array}{l}\text { Chandrapatya \& } \\
\text { Boczek } 1997 b\end{array}$ & Paraxial tibial seta $l$ ' absent. \\
\hline $\begin{array}{l}\text { Vimola terminalis } \\
\text { (Chandrapatya \& Boczek, } \\
\text { 2000) }\end{array}$ & $\begin{array}{l}\text { Rhynacus terminalis } \\
\text { Chandrapatya \& } \\
\text { Boczek, } 2000\end{array}$ & $\begin{array}{l}\text { Chandrapatya \& } \\
\text { Boczek 2000b }\end{array}$ & Paraxial tibial seta $l$ ' absent., \\
\hline \multicolumn{4}{|c|}{ Diptilomiopidae, Rhyncaphytoptinae } \\
\hline $\begin{array}{l}\text { Cathetacarus imperatae } \\
\text { (Chandrapatya, 1993) }\end{array}$ & $\begin{array}{l}\text { Catarhinus imperatae } \\
\text { Chandrapatya, } 1993\end{array}$ & $\begin{array}{l}\text { Chandrapatya \& } \\
\text { Boczek 1993b }\end{array}$ & $\begin{array}{l}\text { Dorsal opisthosoma with a broad, shallow } \\
\text { furrow. }\end{array}$ \\
\hline \multicolumn{4}{|c|}{ New combinations and replacement names } \\
\hline \multicolumn{4}{|c|}{ Eriophyidae, Nothopodinae, Nothopodini } \\
\hline $\begin{array}{l}\text { Cosella trangii Chandrapatya, } \\
\text { Konvipasruang \& Amrine, } \\
2016\end{array}$ & $\begin{array}{l}\text { Aequsomatus } \\
\text { longani Boczek \& } \\
\text { Chandrapatya, } 2000\end{array}$ & $\begin{array}{l}\text { Boczek \& } \\
\text { Chandrapatya 2000c }\end{array}$ & $\begin{array}{l}\text { Belongs to Cosella, with tibia relatively } \\
\text { reduced; internal coxisternal apodeme } \\
\text { absent. The name Cosella longani is too } \\
\text { close to Cosella longana Huang, Huang \& } \\
\text { Wang, 1996; both mites are distinctive and } \\
\text { different; needed a new name. }\end{array}$ \\
\hline \multicolumn{4}{|c|}{ Eriophyidae, Phyllocoptinae, Phyllocoptini } \\
\hline $\begin{array}{l}\text { Phyllocoptes takhoppae } \\
\text { Chandrapatya, Konvipasruang } \\
\text { \& Amrine, } 2016\end{array}$ & $\begin{array}{l}\text { Aequsomatus } \\
\text { indicus Boczek \& } \\
\text { Chandrapatya, } 2000\end{array}$ & $\begin{array}{l}\text { Boczek \& } \\
\text { Chandrapatya 2000c }\end{array}$ & $\begin{array}{l}\text { Belongs to Phyllocoptes because anterior } \\
\text { seta } l b \text { present on coxisternum I. The } \\
\text { name Phyllocoptes indicus was too close } \\
\text { to Phyllocoptes indicae Keifer, } 1975 ; \text { both } \\
\text { mites are distinctive and separate species. } \\
\text { Needed a new name. }\end{array}$ \\
\hline \multicolumn{4}{|l|}{ Replacement names } \\
\hline \multicolumn{4}{|c|}{ Eriophyidae, Phyllocoptinae, Anthocoptini } \\
\hline $\begin{array}{l}\text { Aculops chonburi } \\
\text { Chandrapatya, Konvipasruang } \\
\text { \& Amrine, } 2016\end{array}$ & $\begin{array}{l}\text { Aculops desmodii } \\
\text { Chandrapatya \& } \\
\text { Boczek, } 2000\end{array}$ & $\begin{array}{l}\text { Chandrapatya \& } \\
\text { Boczek 2000a }\end{array}$ & $\begin{array}{l}\text { Name preoccupied by Aculops desmodii } \\
\text { (Keifer, 1964); they are distinctly different. }\end{array}$ \\
\hline \multicolumn{4}{|c|}{ Diptilomiopidae, Diptilomiopinae } \\
\hline $\begin{array}{l}\text { Diptilomiopus sakaena } \\
\text { Chandrapatya, Konvipasruang } \\
\text { \& Amrine, } 2016\end{array}$ & $\begin{array}{l}\text { Diptilomiopus } \\
\text { combretae } \\
\text { (Chandrapatya \& } \\
\text { Boczek, 2002) }\end{array}$ & Craemer et al 2005 & $\begin{array}{l}\text { Name preoccupied by Diptilomiopus } \\
\text { combretae Wei \& Lu, 2001; they are } \\
\text { distinctly different. }\end{array}$ \\
\hline
\end{tabular}




\begin{tabular}{|c|c|c|c|}
\hline New assignment & Original assignment & $\begin{array}{l}\text { Reference for } \\
\text { original assignment }\end{array}$ & Reasons for moving to new genus \\
\hline \multicolumn{4}{|l|}{ Emendations } \\
\hline \multicolumn{4}{|c|}{ Eriophyidae, Cecidophyinae, Cecidophyini } \\
\hline $\begin{array}{l}\text { Cecidophyes ophiopogonis } \\
\text { Keifer, } 1976\end{array}$ & $\begin{array}{l}\text { Cecidophyes } \\
\text { ophiogonis Keifer, } \\
1976\end{array}$ & Keifer 1976 & $\begin{array}{l}\text { Specific name needed to match that of the } \\
\text { host plant genus, Ophiopogon. }\end{array}$ \\
\hline \multicolumn{4}{|c|}{ Eriophyidae, Eriophyinae, Aceriini } \\
\hline -Aceria binaria (Keifer, 1977) & $\begin{array}{l}\text { Eriophyes binarius } \\
\text { Keifer, } 1977\end{array}$ & Keifer 1977b & $\begin{array}{l}\text { Specific name needed to be emended to } \\
\text { binaia, as gender of the genus is feminine }\end{array}$ \\
\hline \multicolumn{4}{|c|}{ Eriophyidae, Phyllocoptinae, Tegonotini } \\
\hline $\begin{array}{l}\text { Shevtchenkella arfeuilleae } \\
\text { Chandrapatya \& Boczek, } \\
2000\end{array}$ & $\begin{array}{l}\text { Schevtchenkella } \\
\text { arfeuilleae } \\
\text { Chandrapatya \& } \\
\text { Boczek, } 2000\end{array}$ & $\begin{array}{l}\text { Chandrapatya \& } \\
\text { Boczek 2000a }\end{array}$ & Generic name was misspelled. \\
\hline $\begin{array}{l}\text {-Tegonotus streblusi Boczek, } \\
1997\end{array}$ & $\begin{array}{l}\text { Tegonotus strebusi } \\
\text { Boczek, } 1997\end{array}$ & $\begin{array}{l}\text { Chandrapatya \& } \\
\text { Boczek 1997a }\end{array}$ & $\begin{array}{l}\text { Specific name needed to beemended to } \\
\text { streblusi after the genus name of the host } \\
\text { plant, Streblus. }\end{array}$ \\
\hline $\begin{array}{l}\text {-Wanleelagus cerberus Boczek, } \\
1997\end{array}$ & $\begin{array}{l}\text { Wanleelagus ceberus } \\
\text { Boczek, } 1997\end{array}$ & $\begin{array}{l}\text { Chandrapatya \& } \\
\text { Boczek } 1997 b\end{array}$ & $\begin{array}{l}\text { Specific name needed to be emended to } \\
\text { cerberus after the genus name of the host } \\
\text { plant, Cerbera. }\end{array}$ \\
\hline \multicolumn{4}{|c|}{ Eriophyidae, Phyllocoptinae, Anthocoptini } \\
\hline $\begin{array}{l}\text {-Tetra minuta (Chandrapatya \& } \\
\text { Boczek, 2000) }\end{array}$ & $\begin{array}{l}\text { Tetra minutus } \\
\text { (Chandrapatya \& } \\
\text { Boczek, 2000) }\end{array}$ & Amrine et al. 2003 & $\begin{array}{l}\text { Specific name needed to be emended } \\
\text { to minuta, as the gender of the genus is } \\
\text { feminine, to match Tetra. }\end{array}$ \\
\hline \multicolumn{4}{|c|}{ Diptilomiopiae, Diptilomiopinae } \\
\hline $\begin{array}{l}\text { Diptilomiopus panithus } \\
\text { (Boczek \& Chandrapatya, } \\
\text { 1989) }\end{array}$ & $\begin{array}{l}\text { Diptilomiopus } \\
\text { pamithus (Boczek \& } \\
\text { Chandrapatya, 1989) }\end{array}$ & Hong \& Zhang 1997 & $\begin{array}{l}\text { Specific name emended to panithus, as per } \\
\text { the name of Mr. Panithan Chandrapatya. }\end{array}$ \\
\hline
\end{tabular}

Junior synonyms

\section{References for original description}

Eriophyidae, Cecidophyinae, Cecidophyini

- Neserella hispidus (Chandrapatya \& Boczek, 2000) ) is a junior synonym of

Neserella capreifoliae Meyer \& Ueckermann, 1989

Eriophyidae, Phyllocoptinae, Calacarini

· Calacarus mellingtoniae Boczek \& Chandrapatya, 1989b) is a junior synonym of Calacarus millingtoniae Mohanasundaram, 1982

Eriophyidae, Phyllocoptinae, Anthocoptini

-Aculodes ventricosae Chandrapatya, 1998 is a synonym of Phaicus bambusae (ChannaBassavanna, 1966; Konvipasruang, 2012a)

-Aculops caricis (Boczek, 1996) is a junior synonym of Aculops caricae Keifer, 1977

-Liparus eugeniae Boczek, 1996 is a junior synonym of Indotegolophus eugeniae Boczek, 1992, nowLipacarus eugeniae (Boczek, 1992) —new genus, new combination

-Tegolophus bangkokensis Keifer, 1978 is a junior synonym of Phaicus bambusae (ChannaBasavanna 1966; Keifer 1978)

-Vasates caricis Boczek, 1996 is a junior synonym of Aculops caricae Keifer, 1977

- Vasates mangiferae Chandrapatya, 1991 is a junior synonym of Metaculus mangiferae (Attiah, 1955)

Amrine et al. 2003

Boczek \& Chandrapatya, 1989b

Boczek \& Chandrapatya, 1996a

Boczek \& Chandrapatya, 1996b

Chandrapatya \& Boczek, 1991c

Diptilomiopidae, Diptilomiopinae

- Diptilomiopus phylanthi (Chandrapatya, 1992) is a junior synonym of Diptilomiopus securinegus Boczek, 1992

Hong \& Zhang, 1997 
Table 3. List of new genera and new combinations assigned to eight previously described eriophyoid mites of Thailand

\begin{tabular}{|c|c|c|}
\hline Original assignment & New assignment & $\begin{array}{l}\text { Reference for original and } \\
\text { new assignment }\end{array}$ \\
\hline \multicolumn{3}{|l|}{ Eriophyidae, Cecidophyinae, Colomerini } \\
\hline Circaces citri Boczek, 1966 & $\begin{array}{l}\text { Makruta citri (Boczek, 1996) } \\
\text { Chandrapatya, Konvipasruang \& Amrine, } \\
2015\end{array}$ & $\begin{array}{l}\text { Boczek \& Chandrapatya } \\
\text { 1996a } \\
\text { Chandrapatya et al. } 2015\end{array}$ \\
\hline \multicolumn{3}{|l|}{ Eriophyidae, Phyllocoptinae, Tegonotini } \\
\hline $\begin{array}{l}\text { Tegonotus suregadi Boczek \& } \\
\text { Chandrapatya, } 2000\end{array}$ & $\begin{array}{l}\text { Khanthongella suregadi (Boczek \& } \\
\text { Chandrapatya, 2000) Chandrapatya, } \\
\text { Konvipasruang \& Amrine, } 2015\end{array}$ & $\begin{array}{l}\text { Boczek \& Chandrapatya } \\
\text { 2000a } \\
\text { Chandrapatya et al. } 2015\end{array}$ \\
\hline $\begin{array}{l}\text { Leipothrix mangiferae (Chandrapatya, } \\
\text { 1997) }\end{array}$ & $\begin{array}{l}\text { Vareeboona mangiferae (Chandrapatya, } \\
\text { 1997) Chandrapatya, Konvipasruang \& } \\
\text { Amrine, } 2015\end{array}$ & $\begin{array}{l}\text { Xue \& Zhang } 2009 \\
\text { Chandrapatya et al. } 2015\end{array}$ \\
\hline \multicolumn{3}{|c|}{ Eriophyidae, Phyllocoptinae, Phyllocoptini } \\
\hline Epitrimerus glutinosae Boczek, 1996 & $\begin{array}{l}\text { Mimenacarus glutinosae (Boczek, 1996) } \\
\text { Chandrapatya, Konvipasruang \& Amrine, } \\
2015\end{array}$ & $\begin{array}{l}\text { Chandrapatya \& Boczek } 1996 \\
\text { Chandrapatya et al. } 2015\end{array}$ \\
\hline \multicolumn{3}{|l|}{ Eriophyidae, Phyllocoptinae, Anthocoptini } \\
\hline Indotegolophus eugeniae Boczek, 1992 & Lipacarus eugeniae (Boczek, 1992) & $\begin{array}{l}\text { Boczek \& Chandrapatya } \\
\text { 1992b } \\
\text { Chandrapatya et al. 2016b }\end{array}$ \\
\hline \multicolumn{3}{|l|}{ Diptilomiopidae, Diptilomiopinae } \\
\hline Acarhis diospyrosis Chandrapatya, 1991 & $\begin{array}{l}\text { Bangkokarus diospyrosis (Chandrapatya, } \\
\text { 1991) Chandrapatya, Konvipasruang \& } \\
\text { Amrine, } 2015\end{array}$ & $\begin{array}{l}\text { Chandrapatya \& Boczek } \\
\text { 1991c } \\
\text { Chandrapatya et al. } 2015\end{array}$ \\
\hline $\begin{array}{l}\text { Diptilomiopus ervatamiae } \\
\text { (Chandrapatya, 1991) }\end{array}$ & $\begin{array}{l}\text { Phutsona ervatamiae (Chandrapatya, } \\
\text { 1991) }\end{array}$ & $\begin{array}{l}\text { Hong \& Zhang } 1997 \\
\text { Chandrapatya et al. } 2016 \mathrm{~b}\end{array}$ \\
\hline \multicolumn{3}{|l|}{ Diptilomiopidae, Rhyncaphytoptinae } \\
\hline $\begin{array}{l}\text { Rhyncaphytoptus talutus Chandrapatya } \\
\text { \& Boczek, } 2000\end{array}$ & $\begin{array}{l}\text { Phayomoca talutus (Chandrapatya } \\
\text { \& Boczek, 2000) Chandrapatya, } \\
\text { Konvipasruang \& Amrine, } 2015\end{array}$ & $\begin{array}{l}\text { Chandrapatya \& Boczek } \\
\text { 2000d } \\
\text { Chandrapatya et al. } 2015\end{array}$ \\
\hline
\end{tabular}

Table 4. List of Eight new genera and ten new species of eriophyoid mites found in Thailand

\begin{tabular}{|c|c|c|}
\hline Scientific names & Systematic arrangement & References \\
\hline $\begin{array}{l}\text { Yangnarus dipterocarpi Chandrapatya, } \\
\text { Konvipasruang \& Amrine, } 2016\end{array}$ & Eriophyidae, Phyllocoptinae, Acaricalini & Chandrapatya et al 2016a \\
\hline $\begin{array}{l}\text { Rapinarus maphoki Chandrapatya, } \\
\text { Konvipasruang \& Amrine, } 2016\end{array}$ & Eriophyidae, Phyllocoptinae, Calacarini & Chandrapatya et al 2016a \\
\hline $\begin{array}{l}\text { Kradukarus suregadi Chandrapatya, } \\
\text { Konvipasruang \& Amrine, } 2016\end{array}$ & $\begin{array}{l}\text { Eriophyidae, Phyllocoptinae, } \\
\text { Phyllocoptini }\end{array}$ & Chandrapatya et al 2016a \\
\hline $\begin{array}{l}\text { Longanella lamyai Chandrapatya, } \\
\text { Konvipasruang \& Amrine, } 2016\end{array}$ & $\begin{array}{l}\text { Eriophyidae, Phyllocoptinae, } \\
\text { Phyllocoptini }\end{array}$ & Chandrapatya et al 2016a \\
\hline $\begin{array}{l}\text { Lamyacarus longani Chandrapatya, } \\
\text { Konvipasruang \& Amrine, } 2016\end{array}$ & $\begin{array}{l}\text { Eriophyidae, Phyllocoptinae, } \\
\text { Anthocoptini }\end{array}$ & Chandrapatya et al 2016a \\
\hline $\begin{array}{l}\text { Khamroka tomentosi Konvipasruang \& } \\
\text { Chandrapatya, } 2015\end{array}$ & Diptilomiopidae, Diptilomiopinae & Konvipasruang \& Chandrapatya, 2015 \\
\hline $\begin{array}{l}\text { Salaya takhronami Konvipasruang \& } \\
\text { Chandrapatya, } 2015\end{array}$ & Diptilomiopidae, Diptilomiopinae & Konvipasruang \& Chandrapatya, 2015 \\
\hline $\begin{array}{l}\text { Wangthonga phayomae Chandrapatya, } \\
\text { Konvipasruang \& Amrine, } 2016\end{array}$ & Diptilomiopidae, Diptilomiopinae & Chandrapatya et al 2016a \\
\hline $\begin{array}{l}\text { Diptilomiopus khatkhaii Konvipasruang } \\
\text { \& Chandrapatya, } 2015\end{array}$ & Diptilomiopidae, Diptilomiopinae & Konvipasruang \& Chandrapatya, 2015 \\
\hline $\begin{array}{l}\text { Diptilomiopus mandongii } \\
\text { Konvipasruang \& Chandrapatya, } 2015\end{array}$ & Diptilomiopidae, Diptilomiopinae & Konvipasruang \& Chandrapatya, 2015 \\
\hline
\end{tabular}


Boczek, 2000 was occupied by Aculops desmodii Keifer (1964). The species epithet is after the place, Chonburi Province, where the mite was collected. Phyllocoptes takhoppae Chandrapatya, Konvipasruang \& Amrine, 2016 for Aequsomatus indicus Boczek \& Chandrapatya, 2000 since the name Phyllocoptes indicus (Boczek \& Chandrapatya, 2000) is too close to Phyllocoptes indicae Keifer, 1975 found in Bangkok, Thailand. The species epithet is after the commone name of the host plant, Takhop. Cosella trangii Chandrapatya, Konvipasruang \& Amrine, 2016 for Aequsomatus longani Boczek \& Chandrapatya, 2000 since the name is too close to Cosella longana Huang, Huang \& Wang, 1996. The species epithet is after the place, Trang Province, where the mite was collected.

\section{CONCLUSION}

Only 2 families of eriophyoid mites, the Eriophyidae (160 species) and Diptilomiopidae (62 species), were found in Thailand. These mites were found in every part of Thailand and infested a wide variety of host plants: 161 host plant species under 60 plant families were recorded. The scientific status of 50 previously described mites was rearranged: 9 species were placed as junior synonyms; 22 new combination names, 2 new combination and replacement names, and 2 replacement names were given to 35 species; and 7 scientific names were emended. Eight new generic names-Bangkokarus, Lipacarus, Phayomoca, Phutsona, Makruta, Mimenacarus, Vareeboona, and Khanthongella - were newly assigned to 8 mites previously identified by the first author. Moreover, 8 new genera and new species namely Lamyacarus longani Chandrapatya, Konvipasruang \& Amrine, 2016, Longanella lamyai Chandrapatya, Konvipasruang \& Amrine, 2016, Khamroka tomentosi Konvipasruang \&Chandrapatya, 2015, Kradukarus suregadi Chandrapatya, Konvipasruang \& Amrine, 2016, Rapinarus maphoki Chandrapatya, Konvipasruang \& Amrine, 2016, Salaya takhronami Konvipasruang \& Chandrapatya, 2015, Wangthonga phayomae Chandrapatya, Konvipasruang \& Amrine, 2016, and Yangnarus dipterocarpi Chandrapatya, Konvipasruang \& Amrine, 2016 and 2 new species: Diptilomiopus khatkhaii and Diptilomiopus mandongii were assigned to unidentified mites in the collection.., .

\section{ACKNOWLEDGEMENTS}

Part of this research was funded by the Faculty of Agriculture and the Center for Advanced Studies for Agriculture and Food, KU Institute for Advanced Studies, Kasetsart University, Bangkok 10900, Thailand (CASAF, NRU-KU, Thailand) and the Thailand Research Fund (TRF Senior Research Scholar \#RTA4880006). We also thank the Central Laboratory, Faculty of Agriculture, Kasetsart University for providing a powerful phase-contrast microscope for use throughout the study.

\section{REFERENCES}

Amrine, J. W. Jr. and T. A. Stasny (1994) Catalog of the Eriophyoidea (Acarina: Prostigmata) of the World. 804 pp., Indira Publish. House, West Bloomfield, Michigan, USA. 
Amrine, J. W. Jr. and T. A. Stasny (1996) Corrections to the catalog of the Eriophyoidea (Acarina: Prostigmata) of the world. International Journal of Acarology, 22: 295-304.

Amrine, J. W. Jr., T. A. Stasny and C. H. W. Flechtmann (2003) Revised Keys to World Genera of Eriophyoidea (Acari: Prostigmata). 244 pp., Indira Publishing House, West Bloomfield, Michigan, USA.

Ashmead, W. H. (1879) Injurious and beneficial insects found on the orange trees in Florida. Canadian Entomologist, 11: $159-160$.

Attiah, H. (1955) A new eriophyid mite on mango from Egypt. Bulletin Societe Entomologique Egypte, 39: 379-383.

Boczek, J. and A. Chandrapatya (1989a) Studies on eriophyid mites (Acari: Eriophyoidea). I. Bulletin of the Polish Academy of Sciences, Biological Sciences, 37: 133-140.

Boczek, J. and A. Chandrapatya (1989b) Studies on eriophyid mites (Acari: Eriophyoidea). II. Bulletin of the Polish Academy of Sciences, Biological Sciences, 37: 141-148.

Boczek, J. and A. Chandrapatya (1992a) Studies on Eriophyid mites (Acari: Eriophyoidea): VI. International Journal of Acarology, 18: 277-285.

Boczek, J. and A. Chandrapatya (1992b) Studies on eriophyid mites (Acari: Eriophyoidea). X. Bulletin of the Polish Academy of Sciences, Biological Sciences, 40: 261-267.

Boczek, J. and A. Chandrapatya (1992c) Studies on Eriophyid mites (Acari: Eriophyoidea). XI. Bulletin of the Polish Academy of Sciences, Biological Sciences, , 40: 269-277.

Boczek, J. and A. Chandrapatya (1996a) Studies on eriophyoid mites (Acari: Eriophyoidea). XVIII. Bulletin of the Polish Academy of Sciences, Biological Sciences, 44: 61-70.

Boczek, J. and A. Chandrapatya (1996b) Studies on eriophyoid mites (Acari: Eriophyoidea). XX. Bulletin of the Polish Academy of Sciences, Biological Sciences, 44: 83-92.

Boczek, J. and A. Chandrapatya (1998a) Studies on eriophyoid mites (Acari: Eriophyoidea). XXII. Acarologia, 39: 135142.

Boczek J. and A. Chandrapatya (1998b) Studies on eriophyoid mites (Acari: Eriophyoidea). XXV. Bulletin of the Polish Academy of Sciences, Biological Sciences, 46: 31-38.

Boczek, J. and A. Chandrapatya (2000a) Studies on eriophyoid mites (Acari: Eriophyoidea). XXX. Bulletin of the Polish Academy of Sciences, Biological Sciences, 48: 135-143.

Boczek, J. and A. Chandrapatya (2000b) Studies on eriophyoid mites (Acari: Eriophyoidea). XXXII. Bulletin of the Polish Academy of Sciences, Biological Sciences, 48: 197-209.

Boczek, J. and A. Chandrapatya (2000c) Studies on eriophyoid mites (Acari: Eriophyoidea). XXXIV. Bulletin of the Polish Academy of Sciences, 48: 228-231, f. 2.

Boczek, J. and A. Chandrapatya (2000d) Studies on eriophyoid mites (Acari: Eriophyoidea). XLI. Bulletin of the Polish Academy of Sciences, Biological Sciences, 48: 351-355, f. 3.

Boczek, J. and A. Chandrapatya (2000e) Studies on eriophyoid mites (Acari: Eriophyoidea). XLIII. Bulletin of the Polish Academy of Sciences, Biological Sciences, 48: 371-382.

Boczek, J. and A. Chandrapatya (2000f) Studies on eriophyoid mites (Acari: Eriophyoidea). XLV. Bulletin of the Polish Academy of Sciences, Biological Sciences, 48: 395-407.

Boczek, J. and A. Chandrapatya (2001) Studies on eriophyoid mites (Acari: Eriophyoidea). XXXVII. Acarologia, 41: 429436.

Boczek, J. and A. Chandrapatya (2002) Studies on Eriophyoid Mites (Acari : Eriophyoidea). XLIX. Bulletin of the Polish Academy of Sciences, Biological Sciences, 50: 25-36.

Boczek, J. and D. Knihinicki (1998) Studies on Eriophyoid Mites. XXVII. Bulletin of the Polish Academy of Sciences, Biological Sciences, 46: 141-146.

Boczek, J. and R. U. Petanovic (1995) Studies on eriophyoid mites (Acari: Eriophyoidea), XVI. Bulletin of the Polish Academy of Sciences, Biological Sciences, 43: 67-75.

Chandrapatya A. and J. Boczek (1991a) Studies on eriophyid mites (Acari: Eriophyoidea). IV. Bulletin of the Polish Academy of Sciences, Biological Sciences, 39: 427-433.

Chandrapatya, A. and J. Boczek (1991b) Studies on eriophyid mites (Acari: Eriophyoidea) V. Bulletin of the Polish Academy of Sciences, Biological Sciences, 39: 435-443. 
Chandrapatya, A. and J. Boczek (1991c) Studies on eriophyid mites (Acari: Eriophyoidea). VIII. Bulletin of the Polish Academy of Sciences, Biological Sciences, 39: 445-452

Chandrapatya, A. and J. Boczek (1993a) Studies on Eriophyid mites (Acari: Eriophyoidea): VII. International Journal of Acarology, 19: 69-73.

Chandrapatya, A. and J. Boczek (1993b) Studies on eriophyid mites (Acari: Eriophyoidea), XII. Bulletin of the Polish Academy of Sciences, Biological Sciences, 41:45-52.

Chandrapatya, A. and J. Boczek (1996) Studies on eriophyoid mites (Acari: Eriophyoidea). XIX. Bulletin of the Polish Academy of Sciences, Biological Sciences, 44: 71-81.

Chandrapatya, A. and J. Boczek (1997a) Studies on eriophyoid mites (Acari: Eriophyoidea). XXI. Bulletin of the Polish Academy of Sciences, Biological Sciences, 45: 11-21.

Chandrapatya, A. and J. Boczek (1997b) Studies on eriophyoid mites (Acari: Eriophyoidea). XXIII. Bulletin of the Polish Academy of Sciences, Biological Sciences, 45: 23-34.

Chandrapatya, A. and J. Boczek (1998) Studies on eriophyoid mites (Acari: Eriophyoidea). XXVI. Bulletin of the Polish Academy of Sciences, Biological Sciences, 46: 39-46.

Chandrapatya, A. and J. Boczek (2000a) Studies on eriophyoid mites (Acari: Eriophyoidea). XXIX. Bulletin of the Polish Academy of Sciences, Biological Sciences, 48: 125-133.

Chandrapatya, A. and J. Boczek (2000b) Studies on eriophyoid mites (Acari: Eriophyoidea). XXXI. Bulletin of the Polish Academy of Sciences, Biological Sciences, 48: 145-155.

Chandrapatya, A. and J. Boczek (2000c) Studies on eriophyoid mites (Acari: Eriophyoidea). XXXIII. Bulletin of the Polish Academy of Sciences, Biological Sciences, 48: 211-223.

Chandrapatya, A. and J. Boczek (2000d) Studies on eriophyoid mites (Acari: Eriophyoidea). XXXVI. Bulletin of the Polish Academy of Sciences, Biological Sciences, 48: 255-267.

Chandrapatya, A. and J. Boczek (2000e) Studies on eriophyoid mites (Acari: Eriophyoidea). XXXVIII. Bulletin of the Polish Academy of Sciences, Biological Sciences, 48: 305-318.

Chandrapatya, A. and J. Boczek (2000f) Studies on eriophyoid mites (Acari: Eriophyoidea). XLII. Bulletin of the Polish Academy of Sciences, Biological Sciences, 48: 359-370.

Chandrapatya, A. and J. Boczek (2000 g) Studies on eriophyoid mites (Acari: Eriophyoidea). XLIV. Bulletin of the Polish Academy of Sciences, Biological Sciences, 48: 383-394.

Chandrapatya, A. and J. Boczek (2001a) Studies on eriophyoid mites (Acari: Eriophyoidea). XLVI. Bulletin of the Polish Academy of Sciences, Biological Sciences, 49: 91-102.

Chandrapatya, A. and J. Boczek (2001b) Studies on eriophyoid mites (Acari: Eriophyoidea). XLVII. Bulletin of the Polish Academy of Sciences, Biological Sciences, 49: 103-114.

Chandrapatya, A. and J. Boczek (2001c) Studies on eriophyoid mites (Acari: Eriophyoidea). XLVIII. Bulletin of the Polish Academy of Sciences, Biological Sciences, 49: 115-126.

Chandrapatya, A and J. Boczek (2002a) Studies on eriophyoid mites (Acari: Eriophyoidea). L. Bulletin of the Polish Academy of Sciences, Biological Sciences, 50: 123-134.

Chandrapatya, A. and J. Boczek (2002b). Studies on eriophyoid mites (Acari: Eriophyoidea). A-1. Bulletin of the Polish Academy of Sciences, Biological Sciences, 50: 135-147.

Chandrapatya, A., P. Konvipasruang and J. W. Amrine, Jr. (2014a) A new genus, two new species, and a new generic name of Phyllocoptine mites (Acari: Eriophyidae) infesting Yang-na, Dipterocarpus alatus Roxb. ex G. Don (Dipterocarpaceae) in Thailand. Journal of Acarological Society of Japan, 23: 15-28.

Chandrapatya, A., P. Konvipasruang, C. H. W. Flechtmann and G. de Moraes (2014b) Complementary description of Colomerus novahebridensis Keifer (Acari, Eriophyidae), with a discussion about the constitution of the genus and its economic importance, and a tentative key to Colomerus Newkirk \& Keifer species. Zookey, 434: 17-35.

Chandrapatya, A., P. Konvipasruang and J. W. Amrine, Jr. (2015) Six new generic names for eriophyoid mites described from Thailand with supplement descriptions and illustrations (Acari: Eriophyoidea). Journal of Systematic and Applied Acarology 20(5): 523-555.

Chandrapatya, A., P. Konvipasruang and J. W. Amrine, Jr. (2016a) New eriophyoid mites from Thailand. Journal of Systematic and Applied Acarology, 21(1): 55-78. 
Chandrapatya, A., P. Konvipasruang and J. W. Amrine, Jr. (2016b) Two new genera and one new assignment of eriophyoid mites (Acari: Eriophyoidea) from Thailand. Persian Journal of Acarology, 5(1): 35-49.

Chandrapatya, A., D. Navia and C. H. W. Flechtmann (2000) Taspinus Chandrapatya, 1991, a junior synonym of Spinacus Keifer, 1979 (Acari: Eriophyidae). International Journal of Acarology, 26: 81-86.

ChannaBasavanna, G. P. (1966) A Contribution to the Knowledge of Indian Eriophyid Mites (Eriophyoidea: Trombidiformes: Acarina). 154 pp., University of Agricultural Sciences, Hebbal, Bangalore, India.

Chetverikov, F. E. (2005) Eriophyid mites (Acari, Eriophyoidea: Eriophyidae) of the genus Leipothrix Keifer, 1966 from sedges (Cyperaceae). Acarina, 13: 145-154.

Craemer, C., J. W. Jr. Amrine, E. de Lillo and T. A. Stasny (2005) Nomenclatural changes and new synonymy in the genus Diptilomiopus Nalepa. 1916 (Acari: Eriophyoidea: Diptilomiopidae). Internaional Journal of Acarology, 31: 134.

Das, B. and S. Chakrabarti (1994) Four new species of phyllocoptine mites (Acari: Eriophyoidea) from northeast India with a discussion on genus Tetra Keifer and key to Indian species. Acarologia, 35: 335-344.

Florida Department of Agriculture and Consumer Services (2006) Quarterly Report No. 2-2006, April 1 to June 30, 2006. Florida Cooperative Agricultural Pest Survey Program, Florida Department of Agriculture and Consumer Services, Division of Plant Industry $26 \mathrm{pp}$. maps.

Ghosh, B., S. Mondal and S. Chakrabarti (1986) Studies on Eriophyid mites (Acarina: Eriophyoidea) of India. Description of three new species from West Bengal. Entomon, 11: 193-198.

Haque, M. M. (2000) Leaf damage in litchi plant caused by Aceria litchi Channabasavanna [(Keifer 1943)] (Acari: Eriophyoidea) and its control. Bangladesh Journal of Zoology, 28: 159-164.

Hong, X.-Y. (1996) Knorella thailandica, a new name for Knorella bambusae Chandrapatya and a key to world species of Knorella (Acari: Eriophyidae). Systematic and Applied Acarology, 1: 205-206.

Hong, X.-Y., D.-S. Wang and Z.-Q. Zhang (2006) Distribution and damage of recent invasive eriophyoid mites (Acari: Eriophyoidea) in Mainland China. International Journal of Acarology, 32: 227-240.

Hong, X.-Y. and Z.-Q. Zhang (1997) Systematics and generic relationships of the mites in the subfamily Diptilomiopinae (Acari: Eriophyoidea: Diptilomiopidae). Systematic Entomology, 22: 313-331.

Huang, T., K.-W. Huang and I.-J. Hong (1989) Two species of eriophyid mites injurious to litchi trees in Taiwan. Chinese Journal of Entomology, Special Publication, 3: 57-64.

Huang T., K.-W. Huang and C.-F. Wang. (1996) Five species of eriophyoid mites of Taiwan: (Acarina: Eriophyoidea: Eriophyidae). Plant Protection Bulletin (Taichung), 38(1): 67-74.

Jeppson, LR. and H. H. Keifer. (1975) Injurious eriophyoid mites. pp. 397-561 In: Jeppson, LR., H. H. Keifer and E. W. Baker. 1975. Mites Injurious to Economic Plants. University of California Press, Berkeley, California, USA. 614 pp.+ 74 plates.

Keifer, H. H. (1938) Eriophyid Studies I. Bulletin of the California Department of Agriculture, 27: 181-206.

Keifer, H. H. (1943) Eriophyid Studies XIII. Bulletin of the California Department of Agriculture, 32: 212-222.

Keifer, H. H. (1944) Eriophyid Studies XIV. Bulletin of the California Department of Agriculture, 33: 18-38.

Keifer, H. H. (1954) Eriophyid studies XXII. Bulletin of the California Department of Agriculture, 43: 121-131.

Keifer H. H. (1955) Eriophyid Studies XXIII. Bulletin of the California Department of Agriculture, 44: 126-130.

Keifer H. H. (1959) Eriophyid Studies XXVII. Occasional Papers. California Department of Agriculture, 2: 1-20.

Keifer, H. H. (1961). Eriophyid Studies B-2. California Department of Food and Agriculture, 1-20.

Keifer, H. H. (1963). Eriophyid Studies B-9. California Department of Food and Agriculture, 1-20.

Keifer, H. H. (1964) Eriophyid Studies B-12. California Department of Food and Agriculture, 1-20.

Keifer, H. H. (1966a) Eriophyid Studies B-18. California Department of Food and Agriculture, 1-20.

Keifer, H. H. (1966b) Eriophyid Studies B-19. California Department of Food and Agriculture, 1-20.

Keifer, H. H. (1966c) Eriophyid Studies B-21. California Department of Food and Agriculture, 1-20.

Keifer, H. H. (1970) Eriophyid Studies C-4. U. S. Department of Agriculture, Agricultural Research Service, 1-24.

Keifer, H. H. (1974) Eriophyoid Studies C-9. U. S. Department of Agriculture, Agricultural Research Service, 1-24.

Keifer, H. H. (1975a) Eriophyid Studies C-10. U. S. Department of Agriculture, Agricultural Research Service, 1-24.

Keifer, H. H. (1975b) Eriophyoid Studies C-11. U. S. Department of Agriculture, Agricultural Research Service, 1-24.

Keifer, H. H. (1976) Eriophyoid Studies C-12. U. S. Department of Agriculture, Agricultural Research Service, 1-24. 
Keifer, H. H. (1977a) Eriophyoid Studies C-13. U. S. Department of Agriculture, Agricultural Research Service, 1-24. Keifer, H. H. (1977b) Eriophyoid Studies C-14. U. S. Department of Agriculture, Agricultural Research Service, 1-24. Keifer, H. H. (1978) Eriophyoid Studies C-15. U. S. Department of Agriculture, Agricultural Research Service, 1-24. Keifer H. H. (1979) Eriophyid Studies C-16. U. S. Department of Agriculture, Agricultural Research Service, 1-24. Keifer, H. H. and L. C. Knorr (1978) Eriophyid Mites of Thailand. Plant Protection Service Technical Bulletin, 38: 1-36. Knihinicki, D. and J. Boczek (2002) New eriophyoid mites (Acari: Prostigmata: Eriophyoidea) from Australia. International Journal of Acarology, 28: 241-249.

Kadono, F. (1994) The occurrence of some species of eriophyid mites injurious to crops in Japan. Shokubutsu Boeki, 487: 294-296.

Konvipasruang, P. and A. Chandrapatya (2015) Two new genera and four new species of Diptilomiopidae from Thailand. Systematic and Applied Acarology, 20(3): 297-312

Konvipasruang, P., A. Chandrapatya and J. W. Amrine Jr. (2012a) Redescription of seven species of eriophyoid mites from bamboo (Poaceae, Bambuseae) in Thailand. Journal of Acarological Society of Japan, 21: 67-94.

Konvipasruang, P., A. Chandrapatya, J. W. Jr. Amrine, R. Ochoa, G. Bauchan and P. Pratt (2012b) A new species, Aceria neopaederiae (Acari: Eriophyidae) infesting Paederia foetida L. (Rubiaceae) in Thailand, Hong Kong and Singapore. Systematic and Applied Acarology, 17: 191-201.

Lakkundi, N. H. (1974) A new species of eriophyid mite, Calacarus channabasavannae (Acarina, Eriophyoidea) from India. Current Research, 3: 70-71.

Lillo de, E. and J. W. Jr. Amrine (2015) Computerized catalog of the Eriophyoidea. In preparation

Maimala S., A.Tartar, D. Boucias and A. Chandrapatya (2002) Detection of the toxin Hirsutellin A from Hirsutella thompsonii. Journal of Invertebrate Pathology, 80: 112-126.

Manson D. C. M. 1973. Two new species of Eriophyid mites (Acarina: Eriophyidae) including a new genus. Acarologia, 15: $96-101$

Manson, D. C. M. and U. Gerson (1996) Web Spinning, Wax Secretion and Liquid Secretion by Eriophyoid Mites. In: Eriophyoid Mites Their Biology, Natural Enemies and Control. (eds., Lindquist, E. E., M. W. Sabelis and J. Bruin), pp. 251-258, Elsevier. World Crop Pests, The Netherlands.

Meyer, M. K. P. (Smith) and E. A. Ueckermann (1989) African Eriophyoidea: a new genus Neserella and Cecidodectes Nalepa (Acari: Eriophyidae) from Trema orientalis (L.) Blume. Phytophylactica, 21: 409-414.

Mohanasundaram, M. (1980) Indian eriophyid studies. II. Mysore Journal of Agricultural Science, 14: 515-528.

Mohanasundaram, M. (1981) New gall-mites of the subfamily Nothopodinae (Acarina; Eriophyidae) from India. Oriental Insects, 15: 145-166

Mohanasundaram, M. (1982) Four new species of Phyllocoptine mites (Eriophiidae: Acarina) from Tamil Nadu. Entomon, 7: 23-30.

Mohanasundaram, M. (1983) Indian eriophyid studies. IV. Record of new phyllocoptine mites (Phyllocoptinae: Eriophyidae: Acarina) from South India. Acarologia, 24: 13-35.

Morse, J. G., R. F. Luck and D. J. Gumpf (1996) Citrus pest problems and their control in the Near East. FAO. Plant Production and Protection Paper, 135: 1-403.

Nalepa, A. (1914) Eriophyiden aus Java (1. Beitrag). Marcellia, 13, 51-87.

Nalepa, A. (1916) Neue Gallmiben (32. Fortsetzung). Anzeiger der kaiserlichen Akademie Wissenschaften. Mathematischenaturwissen-schaftliche Klasse, Wien, 53(22): 283-284.

Nalepa, A. (1917) Neue Gallmiben (32. Fortsetzung). Anzeiger der kaiserlichen Akademie Wissenschaften. Mathematischenaturwissen-schaftliche Klasse, Wien, 54: 52-53.

Ozdikmen, H. (2008) Nomenclatural changes for a family group name and twelve genus group names in Acari. Munis Entomology and Zoology, 3: 211-212.

Roivainen, H. (1951) Contributions to the knowledge of the Eriophyids of Finland. Acta Entomologica Fennica, 8: 1-72.

Sayed, M. T. (1946) Aceria mangiferae nov. spec. Bulletin de la Société Fouad Ier d'Entomologie, 30: 7-10.

Soliman Z. R. and B. A. Abou-Awad. (1978) Five new species of the genus Eriophyes in the A. R. E. (Acarina: Eriophyoidea: Eriophyidae). Acarologia, 19(4): 668-677.

Tan M.-C., L.-F. Huang, Y.-G. Fu and G.-Q Wang (2014) One new genus and two new species of Phyllocoptini (Acari: 
Eriophyidae) from Hainan Island, South China. International Journal of Acarology, 40(7): 513-518.

Wei S.-G. and W. Lu (2001) Two new species of the genus Diptilomiopus (Acari: Diptilomiopidae) from South China. Systematic and Applied Acarology, 6: 115-118.

Xue X.-F., Z. Wang, Z.-W. Song and X.-Y. Hong (2009) Eriophyoid mites on Fagaceae with descriptions of seven new genera and eleven new species (Acari: Eriophyoidea). Zootaxa, 2253: 1-95.

Xue, X. F. and Z. Q. Zhang (2009) Eriophyoid mites (Acari: Prostigmata) in Southeast Asia: a synopsis of 104 genera, with an illustrated key to genera and checklist of species. Zootaxa, 2257: 1-128. 